\title{
Pilot Design for BEM-based Channel Estimation in Doubly Selective Channel
}

\author{
Kushal Anand*, Yong Liang Guan*, Xiaobei Liu*, Zilong Liu**, Yang Yang ${ }^{\dagger}$, Zhengchun Zhou ${ }^{\dagger}$, Pingzhi Fan ${ }^{\dagger}$, Erry Gunawan*
}

\begin{abstract}
This paper investigates pilot design for enhanced channel estimation in single carrier communication systems over doubly-selective channels (DSC). Our contribution is twofold: first, we propose to use Huffman sequences as pilot clusters with low peak-to-average power ratio (PAPR), yet with good channel estimation performance when periodic pilot placement is adopted; second, we propose a low-complexity pilot placement strategy based on the analysis of the complex-exponential basis expansion model (CE-BEM) of the DSC. The latter leads to improved channel estimation performance with useful insights for pilot placement.
\end{abstract}

Index Terms-Doubly-selective channels, Channel estimation, Huffman sequence, Pilot design.

\section{INTRODUCTION}

In recent years, there has been a surge of research interest in high mobility and high rate wireless communications, such as wireless communications for the high speed trains (HSTs) [1]-[4]. These wireless channels tend to suffer from both timeand frequency-selectivity incurred by Doppler and multi-path propagation, respectively, leading to the doubly-selective channels (DSC). Channel estimation (CE) in DSC is a challenging task [1], and is the main focus of this work.

In the literature, various channel modeling and estimation methods have been proposed for the DSC, both in the context of single-carrier [2]-[7] as well as multi-carrier communications [8]-[16]. In many of these works, basis expansion models (BEM) have been used to parameterize the channels which can be represented as a weighted combination of only a few basis functions [13]. The basis functions are already known to the receiver, and therefore it needs to estimate the corresponding BEM coefficients only. Since only few BEM

Copyright(c) 2015 IEEE. Personal use of this material is permitted. However, permission to use this material for any other purposes must be obtained from the IEEE by sending a request to pubs-permission@ieee.org. This work was supported by the NRF-NSFC project under Grant NRF2016NRF-NSFC001-089. The works of Yong Liang Guan and Xiaobei Liu was also supported by Temasek Laboratories@NTU Signal Research Programme 3 Grant. The work of Z. Liu was also supported in part by National Natural Science Foundation of China (Grant No. 61750110527), Research Fund for International Young Scientists. The work of Zhengchun Zhou was supported in part by the Application Fundamental Research Plan Project of Sichuan Province under Grant 2018JY0046. The work of Pingzhi Fan was supported by NSFC Project (No.61661146003 and No.61731017) and 111 Project (No.111-2-14). Kushal Anand, Yong Liang Guan, Xiaobei Liu and Erry Gunawan are with the School of Electrical and Electronic Engineering, Nanyang Technological University, Singapore (email: kush0005@e.ntu.edu.sg, \{eylguan,xpliu,egunawan\}@ntu.edu.sg). Zilong Liu is with the Institute for Communication Systems, Univ. of Surrey, UK (email:zilong.liu@surrey.ac.uk). Yang Yang, Zhengchun Zhou and Pingzhi Fan are with the Southwest Jiaotong University, Chengdu, China (email:\{yang_data,zzc,pzfan\}@ @ swjtu.edu.cn). coefficients need to be estimated, the number of pilot symbols required for $\mathrm{CE}$ is significantly reduced, thereby increasing the spectral efficiency. Thus, the BEM-based approach greatly simplifies the CE complexity compared to the case when we need to estimate the channel coefficient at every time/frequency instant.

Different types of BEMs have been used to model the time-varying channels, for example, the conventional complex-exponential BEM (CE-BEM) [2], generalized CEBEM (GCE-BEM) [3], non-critically sampled CE-BEM (NCSCE-BEM) [17], polynomial BEM [14], [18]-[20], discrete prolate spheroidal BEM (DPS-BEM) [6], [21], and KarhunenLoeve BEM (KL-BEM) [22]. Among all these, KL-BEM provides the most accurate modeling but requires the knowledge of channel statistics which also needs to be periodically updated for the fast time-varying (high Doppler) channels. All other BEMs are independent of channel statistics, and therefore, may be more suited for modeling the time-varying channels. For example, P-BEM is particularly attractive because of its low modeling errors, especially in the low-Doppler cases [18]. Notably, CE-BEM and its variants, GCE-BEM and NCS-CE$\mathrm{BEM}$ are also widely used due to their analytical tractability [23]. We consider NCS-CE-BEM because of its good channel modeling with less number of BEM coefficients for high Doppler scenarios, as well as due to its ease of tractability.

In the literature, various techniques have been proposed for CE in DSC, which comprise pilot-aided, blind as well as semi-blind estimation methods. Blind or semi-blind CE techniques, respectively, require no or minimum pilots for $\mathrm{CE}$, resulting in greater spectral efficiency (SE) as compared to the pilot-aided techniques [24]. Recently, reliable data-aided virtual pilot based semi-blind $\mathrm{CE}$ technique has been reported in [25] which uses the soft symbol decisions to iteratively refine the CE quality. Further, an interesting one-shot blind $\mathrm{CE}$ has been proposed in [26] for OFDM transmission which exploits different modulation types on different subcarriers to obtain high CE accuracy and low receiver compelxity over quasi-static multi-path channels such as that mentioned in [27]. However, in general, blind and semi-blind CE methods are found to work well in slowly varying channels and may incur high receiver complexity [28]. On the other hand, pilot-aided techniques have comparatively low SE but they are extremely useful to estimate or track the fast time-varying channels where the channel varies for each symbol in the transmission block, and are therefore widely used in the current wireless standards such as LTE-A, 802.11p/n etc. 
In this paper ${ }^{1}$, we address pilot-aided $\mathrm{CE}$ for single-carrier (SC) communication over DSC. In most of the existing works on pilot-aided SC transmission, impulse-based pilots or timedomain Kronecker-delta ("TDKD") pilot clusters have been employed for CE. Illustration of the TDKD pilot clusters can be seen in Fig. 1. These pilots, however, suffer from high PAPR during transmission which could lead to inefficient transmission [30], as described next. Since there are very few impulse pilots (with zero padding on both sides of the nonzero pilot) in a transmission block, high power is given to the pilots to obtain a reasonably good channel estimate. For example, as high as $35 \%-50 \%$ of the total transmission power should be allocated to the pilots to obtain the optimal CE and maximum system capacity [2]. This leads to very high power per pilot symbol, which in turn, can drive the transmitter power amplifier into its non-linear operating region, thus degrading the CE quality. Notice that PAPR problem may arise in SC systems due to other reasons such as modulation and pulseshaping [31], but here we consider the PAPR problem arising from the impulse-based TDKD pilot structure.

We address the above problem by proposing a sequencebased pilot scheme where the training power is distributed over multiple sequence symbols (instead of a single non-zero symbol as in the TDKD pilot structure), leading to low PAPR pilot transmission. However, while spreading the pilot power over a sequence, we need to ensure that the zero out-of-phase aperiodic auto-correlation (OP-AAC) property of the impulse pilots is preserved so as to obtain a good CE quality. Note that periodically-placed clustered pilots or sequences have been investigated earlier for the multi-carrier systems under rapidly time-varying channels [32]-[35]. However, our work is very different from the existing works because we intend to use a specific type of sequence with zero OP-AAC property to satisfy a particular CE optimality condition for single-carrier DSC. Moreover, based on our analysis, we have also optimized the pilot positions to improve the CE quality.

First, we analyse the CE mean square error (CE MSE) for SC transmission in detail, and show that good quality $\mathrm{CE}$ can be obtained by using sequences with zero OP-AAC property, together with appropriate placement of the pilots within the transmission block. Using proper sequences and suitable pilot placement, respectively, we can reduce two types of interferences during the BEM coefficients' estimation, namely, the inter-path interference and the inter-BEM frequency interference terms, which improves the CE quality significantly.

Second, to satisfy the zero OP-AAC criterion, we specifically propose the use of Huffman sequences as potential pilot sequences. Huffman sequences are appealing, not only because of their excellent AAC property, i.e., zero OP-AAC for all except the last shift, but also because they offer flexible PAPRs while still maintaining zero OP-AAC. Thus, we can choose a Huffman sequence with low PAPR and yet having zero OPAAC for a required number of time shifts. Furthermore, Huff-

\footnotetext{
${ }^{1}$ Part of this work has been presented in the conference paper [29]
}

man sequences can be systematically generated by efficient algorithms [36], [37].

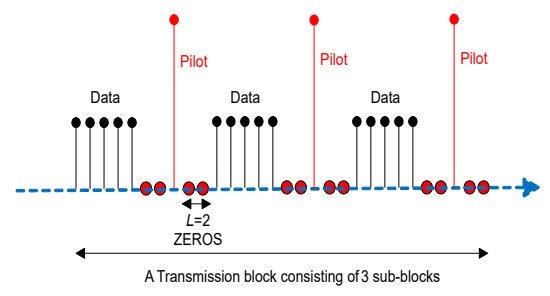

Fig. 1: A transmission block consisting of three sub-blocks, each containing a timedomain Kronecker delta (TDKD) pilot cluster. Each impulse pilot is surrounded by $L$ zeros on both sides, where $L$ denotes the number of multi-paths.

Third, based on the CE MSE analysis, we propose a pilot placement strategy to reduce the inter-BEM frequency interference during BEM coefficients' estimation, thus leading to significantly improved CE performance. In the past, several pilot placement strategies have been proposed but these methods generally suffer from prohibitively high computational complexity [38]. For example, a genetic algorithm based pilot positioning strategy has been proposed in [38] whereas a heuristic strategy has been mentioned in [17]. In contrast to these works, our proposed method uses a low-complexity optimization without any complex matrix inversion operations. Also, our proposed strategy is based on a systematic analysis of the CE MSE, and unveils important connections between the CE MSE and the pilot design process.

The rest of the organization of the paper is as follows. In Section II, we introduce the system model of data transmission and the channel model for DSC. In Section III, we describe the sequence pilot structure and the estimation of the BEM coefficients for the DSC. In Section IV, we analyze the channel estimation in detail, and provide a simplified approximation of the channel estimation error. Based on the analysis in Section IV, we propose the Huffman sequence based pilot design and a low-complexity pilot placement strategy in Section V. Simulation results are provided in Section VI and conclusion is given in Section VII.

Notations: Throughout the paper, upper and lower bold face letters denote matrix and vector, respectively. $\mathbf{X}^{H}, \mathbf{X}^{T}$ and $\mathbf{X}^{*}$ denote the conjugate transpose, transpose and the conjugate of the matrix $\mathbf{X}$, respectively. $\mathbf{X}^{-1}$ denotes the inverse operation on the square matrix $\mathbf{X} .[\mathbf{X}]_{(i, j)}$ denotes the $(i, j)$-th element of the matrix $\mathbf{X} . \operatorname{tr}[\mathbf{X}]$ denotes the trace of the matrix $\mathbf{X}$. $[\mathbf{X}]_{(i,:)}$ and $\mathbf{x}_{i}$ denote the $i^{t h}$ row of the matrix $\mathbf{X}$, and the $i^{t h}$ element of the vector $\mathbf{x}$, respectively. $\mathbb{E}\{\cdot\}$ is the expectation operator. $\mathbf{I}_{N}, \mathbf{0}_{M \times N}$ and $\mathbf{0}_{M}$ denote the $N \times N$ identity matrix, a zero matrix of dimension $M \times N$ and a length$M$ zero vector, respectively. $\mathcal{C N}(0, x)$ denotes a circularly symmetric complex normal variable with mean 0 and variance $x .(a)^{+}=\max (a, 0)$.

\section{System And Channel Model}

\section{A. System Model}

We consider transmission over a DSC with a single antenna each at the transmitter and the receiver. A block-based trans- 
mission design is adopted, where the data-pilot multiplexed symbols (see Fig. 1) are transmitted and received in blocks of length $N$, i.e., $[x[1], x[2], \ldots, x[N]]^{T}$, where $x[n]$ denotes the transmitted symbol at the $n^{\text {th }}$ time-instant. The transmitter sends the data $x[n]$ at a data rate of $\frac{1}{T}$ symbols/sec, where $T$ denotes the symbol time. Then the discrete-time baseband representation of the received signal $y[n]$ is given as

$$
y[n]=\sum_{l=0}^{L} h[n ; l] x[n-l]+v[n],
$$

where $h[n ; l]$ denotes the digital baseband time-varying multipath channel impulse response (CIR), which subsumes the physical channel between the transmitter and the receiver, the transmit as well as the receive pulse shaping filters, at the $n^{\text {th }}$ time instant for an impulse introduced $l$ samples previously, $L$ denotes the number of multi-paths defined as $L=\left\lfloor\frac{\tau_{\max }}{T}\right\rfloor$, with $\tau_{\max }$ being the maximum delay spread of the channel, and $v[n]$ denotes the circularly symmetric AWGN, with statistical distribution of $\mathcal{C N}\left(0, \sigma_{v}^{2}\right)$.

\section{B. Channel Model}

A DSC can be represented in the continuous time $(t)$ $\operatorname{delay}(\tau)$ domain as

$$
h(t ; \tau)=\int_{-\infty}^{\infty} S_{\mathbf{H}}(\tau, \nu) e^{j 2 \pi t \nu} d \nu,
$$

where $S_{\mathbf{H}}(\tau, \nu)$ denotes the spreading function of the channel in the delay $(\tau)$-Doppler $(\nu)$ domain [39]. The digital baseband version of the CIR $h[n ; l]$ (see (1)) can then be modeled using the CE-BEM [2] or its variants such as NCS-CE-BEM [17], where the channel at the $l^{\text {th }}$ delay tap and the $n^{\text {th }}$ time-instant is expressed as a combination of a few complex exponential basis functions, i.e.,

$$
h[n ; l]=\sum_{q=0}^{Q} h_{q}(l) e^{j \omega_{q} n}+e[n],
$$

where $\omega_{q}$ denotes the $q^{\text {th }}$ BEM modeling frequency, $h_{q}(l)(q \in$ $\{0,1, \ldots, Q\})$ the $q^{\text {th }}$ weight or the $q^{\text {th }}$ BEM coefficient ${ }^{2}$ corresponding to the $l^{\text {th }}(l=1, \ldots, L)$ path, $Q:=2\left\lceil f_{\max } N T\right\rceil$ the number of BEM coefficients, and $f_{\max }$ the maximum Doppler frequency. The modeling error is denoted by $e[n]$, and can be minimized by choosing the appropriate basis functions. For the above mentioned DSC representation, we choose the NCS-CE-BEM with the $(Q+1)$ BEM frequencies $\omega_{q}$ 's uniformly distributed between $\left[-2 \pi f_{\max } T,+2 \pi f_{\max } T\right]$ [17], because it provides a much lower modeling error as compared to the critically-sampled CE-BEM in [2]. Notice that NCS-CE-BEM is also a variant of CE-BEM but its modeling frequencies are constrained within the Doppler range $\pm f_{\max }$, thus leading to better channel modeling.

2 It is worth noting here that $h_{q}(l)$ in (3) corresponds to a discretized version of the spreading function $S_{\mathbf{H}}(\tau, \nu)$ in (2). Please refer to [40] for discrete delay-Doppler spreading function.

\section{Pilot StRucture AND ChanNel ESTIMATION}

\section{A. Pilot Structure}

We assume that the data-pilot multiplexed $k^{\text {th }}$ transmitted block consists of $P$ sub-blocks, with the $p^{t h}$ sub-block consisting of a data vector sub-block $\mathbf{s}_{p}$ and a pilot vector sub-block $\mathbf{b}_{p}$, and is given as

$$
\mathbf{x} \triangleq\left[\mathbf{s}_{1}^{T}(k), \mathbf{b}_{1}^{T}(k), \ldots, \mathbf{s}_{P}^{T}(k), \mathbf{b}_{P}^{T}(k)\right]^{T}, \forall k .
$$

Specifically, the pilot cluster in the $p^{\text {th }}$ sub-block can be written as

$$
\begin{aligned}
\mathbf{b}_{p} & =[\underbrace{b_{p, 0}, \ldots, b_{p,(L-1)}}_{\text {set to zeros }}, b_{p, L}, \ldots, b_{p, L+M-1}, \underbrace{b_{p, L+M \ldots, b_{p,\left(N_{p}-1\right)}}}_{\text {set to zeros }}]^{T} \\
& =\left[0,0, \ldots 0,, b_{p, L}, \ldots, b_{p, L+M-1}, 0,0, \ldots 0\right]^{T},
\end{aligned}
$$

where the first and the last $L$ elements of the pilot cluster are set to zeros (as shown above) so as to avoid the inter-symbol interference between the data and the pilot symbols across the sub-block and the main block [2]. The length of each pilot cluster is $N_{p}=2 L+M$, where $M$ is the length of the pilot sequence we wish to design, $2 L$ comes from the zeros on both sides of the sequence. For "impulse" pilot, $M=1$ and $N_{p}=2 L+1$.

Unlike [2] and other existing works, the non-zero pilot symbols in $\mathbf{b}_{p}$, i.e., $\left[b_{p, L}, \ldots, b_{p, L+M-1}\right]$ is a sequence of length $M$, instead of an impulse. An example of such pilot sequence has been shown in Fig. 2. The motivation behind using a sequence as pilot is to reduce the PAPR of the transmitted pilot. Formally, the PAPR of a time-domain length- $N$ sequence b with elements $\left\{b_{n}\right\}$ is defined as [41]:

$$
\rho(\mathbf{b})=\frac{\max _{0 \leq n \leq N-1}\left|b_{n}\right|^{2}}{(1 / N) \sum_{n=0}^{N-1}\left|b_{n}\right|^{2}}
$$

From (5), the PAPR of the impulse pilot shown in Fig. 1 is $2 L+1$. Clearly, by spreading the pilot power over multiple elements of a sequence, we can reduce the pilot PAPR. The challenge here is that how to design low-PAPR pilot sequence while there is no performance loss in channel estimation as compared to the impulse pilot based scheme. To this end, we propose to use Huffman sequences as the pilot clusters, which will be detailed in Section IV.

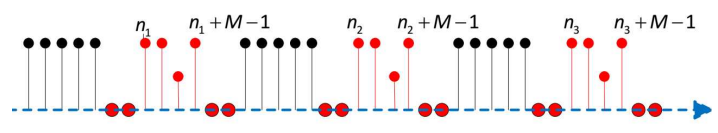

Fig. 2: A transmission block consisting of three sub-blocks, each containing a data subblock (black) and a sequence-based pilot cluster (red). Each sequence pilot is surrounded by $L=2$ zeros on both sides, where $L$ denotes the number of multi paths. $n_{i}$ denotes the "start" position of the length- $M$ sequence in the $i^{t h}$ sub-block.

\section{B. Estimation of BEM Coefficients [2]}

Since the DSC is modeled using BEM, we only need to estimate the BEM coefficients corresponding to appropriate 
basis functions in order to construct the channel at every time instant. Using (1) and (3), the received signal can be written in matrix form as

$$
\mathbf{y}=\mathbf{H x}+\mathbf{v},
$$

where $\mathbf{H}=\sum_{q=0}^{Q} \mathbf{D}_{q} \mathbf{H}_{q}, \mathbf{D}_{q}=\operatorname{diag}\left[1, e^{j \omega_{q}}, \ldots, e^{j \omega_{q}(N-1)}\right]$, and $\mathbf{H}_{q}$ is a lower triangular Toeplitz matrix with first column $\left[h_{q}(0), \ldots, h_{q}(L), 0, \ldots, 0\right]^{T}$, and $\mathbf{v}$ consists of the AWGN components, i.e., $v[n]$ defined in Section II-A. Due to the zero-padding on both sides of the pilot sequence, (6) can be segregated into separate data and the pilot equations $\left(\mathbf{y}_{s}\right.$ and $\mathbf{y}_{b}$, respectively) with corresponding channel matrices $\mathbf{H}_{s}$ and $\mathbf{H}_{b}$, and noise vectors $\mathbf{v}_{s}$ and $\mathbf{v}_{b}$, where

$$
\begin{aligned}
\mathbf{y}_{s} & =\mathbf{H}_{s} \mathbf{s}+\mathbf{v}_{s}, \\
\mathbf{y}_{b} & =\mathbf{H}_{b} \mathbf{b}+\mathbf{v}_{b},
\end{aligned}
$$

with $\mathbf{s} \triangleq\left[\mathbf{s}_{1}^{T}, \ldots, \mathbf{s}_{P}^{T}\right]$, and $\mathbf{b} \triangleq\left[\mathbf{b}_{1}^{T}, \ldots, \mathbf{b}_{P}^{T}\right]^{T}$.

Focusing on channel estimation, we can write (8) as:

$$
\mathbf{y}_{b}=\sum_{q=0}^{Q}\left[\begin{array}{c}
\mathbf{D}_{q, 1} \mathbf{H}_{q, 1} \mathbf{b}_{1} \\
\vdots \\
\mathbf{D}_{q, P} \mathbf{H}_{q, P} \mathbf{b}_{P}
\end{array}\right]+\mathbf{v}_{b},
$$

where $\mathbf{D}_{q, p}$ and $\mathbf{H}_{q, p}$ correspond to the sub-matrices corresponding to the pilot symbols obtained from $\mathbf{D}_{q}$ and $\mathbf{H}_{q}$ (shown below (6)), respectively. Specifically, $\mathbf{D}_{q, p}(q \in$ $\{0,1, \ldots, Q\}, p \in\{1,2, \ldots, P\})$ for the $q^{t h}$ BEM frequency and the $p^{t h}$ sub-block can be written as

$$
\mathbf{D}_{q, p}=\operatorname{diag}\left(\left[e^{j \omega_{q}\left(n_{p}\right)}, e^{j \omega_{q}\left(n_{p}+1\right)}, \ldots, e^{j \omega_{q}\left(n_{p}+N_{p}-L-1\right)}\right]\right),
$$

where $n_{p} \in\{1, \ldots, N\}$ denotes the start position of the $p^{t h}$ non-zero pilot symbol in a transmission block (see Fig. 2).

Next, define the pilot matrix $\mathbf{B}_{p}$ as

$$
B_{p} \triangleq\left[\begin{array}{ccc}
b_{p, L} & \cdots & b_{p, 0} \\
\vdots & \ddots & \vdots \\
b_{p, N_{p}-1} & \cdots & b_{p, N_{p}-L-1}
\end{array}\right],
$$

It can be verified that $\mathbf{H}_{q, p} \mathbf{b}_{p}=\mathbf{B}_{p} \mathbf{h}_{q}$ [2]. Denoting $\mathbf{h}_{q} \triangleq$ $\left[h_{q}(0), \ldots, h_{q}(L)\right]^{T}$, and $\mathbf{h} \triangleq\left[\mathbf{h}_{0}^{T}, \ldots, \mathbf{h}_{Q}^{T}\right]^{T}$, we can write the pilot equation (9) as

$$
\mathbf{y}_{b}=\mathbf{\Phi} \mathbf{h}+\mathbf{v},
$$

where $\boldsymbol{\Phi}$ is defined as

$$
\left[\begin{array}{ccc}
\mathbf{D}_{0,1} \mathbf{B}_{1} & \cdots & \mathbf{D}_{Q, 1} \mathbf{B}_{1} \\
\vdots & \ddots & \vdots \\
\mathbf{D}_{0, P} \mathbf{B}_{P} & \cdots & \mathbf{D}_{Q, P} \mathbf{B}_{P}
\end{array}\right]
$$

From (12), the MSE-based estimate of the BEM coefficient vector $\widehat{\mathbf{h}}$ is given as

$$
\widehat{\mathbf{h}}=\left(1 / \sigma_{v}^{2}\right)\left(\boldsymbol{\Gamma}^{-1}+\left(1 / \sigma_{v}^{2}\right) \boldsymbol{\Phi}^{H} \boldsymbol{\Phi}\right)^{-1} \boldsymbol{\Phi} \mathbf{y}_{b},
$$

where the BEM correlation matrix $\boldsymbol{\Gamma}=\mathbb{E}\left\{\mathbf{h} \mathbf{h}^{H}\right\}$ is assumed to be known at the receiver. The MSE of the BEM coefficients' estimation (referred to as "BEM-MSE") is then given as

$$
M S E \triangleq \operatorname{tr}\left(\left(\boldsymbol{\Gamma}^{-1}+\left(1 / \sigma_{v}^{2}\right) \boldsymbol{\Phi}^{H} \boldsymbol{\Phi}\right)^{-1}\right) .
$$

Note that for a reasonably good BEM representation of the DSC, the modeling error can be neglected, and thus minimizing BEM-MSE also minimizes the actual channel estimation MSE. Then, to minimize the BEM-MSE shown in (15), we need to design the pilot sequences $\mathbf{b}_{p}$ (or $\mathbf{B}_{p}$ ) so that the MSE matrix $\boldsymbol{\Phi}^{H} \boldsymbol{\Phi}$ is diagonal or close to diagonal [42]. It has been shown in [2] that $\boldsymbol{\Phi}^{H} \boldsymbol{\Phi}$ will be diagonal if periodic placement of impulse pilot is adopted, the transmission block length $N$ is an integer multiple of the number of sub-blocks, and the DSC can be accurately represented using CE-BEM with BEM frequencies $\omega_{q}$ 's uniformly distributed between $\left[\frac{2 \pi}{N}\left(\frac{-Q}{2}\right), \frac{2 \pi}{N}\left(\frac{Q}{2}\right)\right]$, i.e., $\omega_{q}=\frac{2 \pi}{N}\left(q-\frac{Q}{2}\right), q \in\{0,1, \ldots, Q\}$. However, for practical wireless channels, the conventional CE-BEM may not be able to model the channel accurately, resulting in large modeling errors [4] and poor CE even when the matrix $\boldsymbol{\Phi}^{H} \mathbf{\Phi}$ is completely diagonal.

More accurate BEM modeling of the channels has been reported by choosing suitable BEM basis functions [3], [6], [17], [21] to improve the CE in practical channels. However, using the above mentioned BEM modeling approaches, the off-diagonal elements of $\boldsymbol{\Phi}^{H} \boldsymbol{\Phi}$ may still be non-zeros. Thus, further $\mathrm{CE}$ improvement is possible by minimizing the offdiagonal elements. In the next sections, we address this issue by analyzing and designing pilot sequences such that the offdiagonal elements of $\boldsymbol{\Phi}^{H} \boldsymbol{\Phi}$ are reduced in magnitude, possibly to a very small value, thus leading to better CE.

\section{Channel estimation analysis}

We can expand $\boldsymbol{\Phi}^{H} \boldsymbol{\Phi}$ as:

$$
\left[\begin{array}{cccc}
\sum_{i=1}^{P} \mathbf{B}_{i}^{H} \mathbf{D}_{0 i}^{H} \mathbf{D}_{0 i} \mathbf{B}_{i} & \sum_{i=1}^{P} \mathbf{B}_{i}^{H} \mathbf{D}_{0 i}^{H} \mathbf{D}_{1 i} \mathbf{B}_{i} & \cdots & \sum_{i=1}^{P} \mathbf{B}_{i}^{H} \mathbf{D}_{0 i}^{H} \mathbf{D}_{Q i} \mathbf{B}_{i} \\
\sum_{i=1}^{P} \mathbf{B}_{i}^{H} \mathbf{D}_{1 i}^{H} \mathbf{D}_{0 i} \mathbf{B}_{i} & \sum_{i=1}^{P} \mathbf{B}_{i}^{H} \mathbf{D}_{1 i}^{H} \mathbf{D}_{1 i} \mathbf{B}_{i} & \cdots & \sum_{i=1}^{P} \mathbf{B}_{i}^{H} \mathbf{D}_{1 i}^{H} \mathbf{D}_{Q i} \mathbf{B}_{i} \\
\vdots & \vdots & \ddots & \vdots \\
\sum_{i=1}^{P} \mathbf{B}_{i}^{H} \mathbf{D}_{Q i}^{H} \mathbf{D}_{0 i} \mathbf{B}_{i} & \sum_{i=1}^{P} \mathbf{B}_{i}^{H} \mathbf{D}_{Q i}^{H} \mathbf{D}_{1 i} \mathbf{B}_{i} & \cdots & \sum_{i=1}^{P} \mathbf{B}_{i}^{H} \mathbf{D}_{Q i}^{H} \mathbf{D}_{Q i} \mathbf{B}_{i}
\end{array}\right],
$$

It is noted that $\boldsymbol{\Phi}^{H} \mathbf{\Phi}$ consists of $(Q+1)$ rows, with each row consisting of $(Q+1)$ sub-matrices, each of size $(L+$ $1) \times(L+1)$. From (16), diagonalizing $\boldsymbol{\Phi}^{H} \boldsymbol{\Phi}$ is equivalent to solving the following two equations:

$$
\begin{aligned}
& \sum_{i=1}^{P} \mathbf{B}_{i}^{H} \mathbf{D}_{j i}^{H} \mathbf{D}_{j i} \mathbf{B}_{i}=\sum_{i=1}^{P} \mathbf{B}_{i}^{H} \mathbf{B}_{i}=\mathcal{P} \mathbf{I}, \\
& \sum_{i=1}^{P} \mathbf{B}_{i}^{H} \mathbf{D}_{q_{1} i}^{H} \mathbf{D}_{q_{2} i} \mathbf{B}_{i}=0, q_{1} \neq q_{2},
\end{aligned}
$$

where $\mathcal{P}$ denotes the power allocated to the pilot sequence in one transmission block. Next, we provide an analysis of the pilot design and the physical interpretations of equations (17) and (18) which will be useful for the design of low-complexity pilot structure. 


\section{A. Analysis of Pilot Equations for CE}

In this section, we provide an approach to estimate the BEM coefficients, and show its connection to the diagonality of the $\boldsymbol{\Phi}^{H} \boldsymbol{\Phi}$ matrix (equivalently, (17) and (18) will be satisfied). We assume that the pilot sequences used in all the $P$ sub-blocks of the transmission block are the same, i.e., $\mathbf{b}_{p}=\mathbf{b}, p=1, \ldots, P$, or equivalently, $b_{p, j}=b_{j}$ in (4). Furthermore, let us assume that the first non-zero symbol of the pilot sequence in the $p^{t h}$ sub-block starts at the $n_{p}$-th position, $n_{p}$ being a positive integer. To focus on the interfering terms for the estimation of the BEM coefficients, in the next analysis, we neglect the noise terms. Then, using (1) and (3) (with $e[n]$ set to zero assuming accurate BEM modeling), the received signal corresponding to the pilot sequence at the $\left(n_{p}+i\right)^{t h}$ position $(i=0, \ldots, M+$ $L-1)$ can be written as

$$
y\left(n_{p}+i\right)=\sum_{l=0}^{i} b_{i-l+L} \sum_{q=0}^{Q} h_{q}(l) e^{j \omega_{q}\left(n_{p}+i\right)} .
$$

Note that in (19), $h_{q}(l)=0$ for $l>L$ because of the finite channel memory. Also, $b_{i-l+L}=0$ for $(i-l)>(M-1)$ because the sequence length is $M$ (see (4)). Collecting the pilot equations from all the sub-blocks, corresponding to $\left(n_{p}+i\right)^{t h}(p=1, \ldots, P)$ position, we have

$$
\begin{aligned}
& \mathbf{y}(i)=\left[y\left(n_{1}+i\right) y\left(n_{2}+i\right) \ldots y\left(n_{P}+i\right)\right]^{T} \\
& =\mathbf{F h}(0) b_{i+L}+\mathbf{F h}(1) b_{i-1+L}+\ldots+\mathbf{F h}(i) b_{L},
\end{aligned}
$$

where $\mathbf{F}$ is defined as

$$
\mathbf{F} \triangleq\left[\mathbf{f}_{0}(i), \ldots, \mathbf{f}_{Q}(i)\right]=\left[\begin{array}{ccc}
e^{j \omega_{0}\left(n_{1}+i\right)} & \ldots & e^{j \omega_{Q}\left(n_{1}+i\right)} \\
e^{j \omega_{0}\left(n_{2}+i\right)} & \ldots & e^{j \omega_{Q}\left(n_{2}+i\right)} \\
\vdots & \ddots & \vdots \\
e^{j \omega_{0}\left(n_{P}+i\right)} & \ldots & e^{j \omega_{Q}\left(n_{P}+i\right)}
\end{array}\right]
$$

with $\mathbf{f}_{q}(i) \triangleq\left[e^{j \omega_{q}\left(n_{1}+i\right)}, \ldots, e^{j \omega_{q}\left(n_{P}+i\right)}\right]^{T}$, and $\mathbf{h}(l) \triangleq$ $\left[h_{0}(l), h_{1}(l), \ldots, h_{Q}(l)\right]^{T}$ is a vector consisting of the BE$\mathrm{M}$ coefficients corresponding to all the BEM frequencies $\omega_{q},(q=0,1, \ldots, Q)$ for the $l^{\text {th }}$ path.

Now, we focus on estimating the BEM coefficient corresponding to the $q^{\text {th }}$ BEM frequency for the $l^{\text {th }}$ path, i.e., $h_{q}(l)$. Multiplying both sides of $\mathbf{y}(i),(i=0, \ldots,(M-1))$ by $\mathbf{f}_{q}^{H}(i)$, we obtain

$$
\begin{aligned}
\mathbf{f}_{q}^{H}(i) \mathbf{y}(i) & =P h_{q}(l) b_{i+L}+\underbrace{P \sum_{l^{\prime} \neq l}^{i} h_{q}\left(l^{\prime}\right) b_{i-l+L}}_{I P I} \\
& +\underbrace{\sum_{l^{\prime}} b_{i-l^{\prime}+L} \sum_{q^{\prime} \neq q} \mathbf{f}_{q}(i)^{H} \mathbf{f}_{q^{\prime}}(i) h_{q^{\prime}}\left(l^{\prime}\right)}_{I B I} .
\end{aligned}
$$

In (22), IPI, referred to as inter-path interference, denotes the interference to $h_{q}(l)$ from the BEM coefficients of the undesired paths but the same BEM frequency $q$, i.e., $h_{q}\left(l^{\prime}\right), l^{\prime} \neq l$. IBI, referred to as inter-BEM-frequency interference, denotes the interference to $h_{q}(l)$ from different BEM frequencies of all the paths, i.e., $h_{q^{\prime}}\left(l^{\prime}\right), q^{\prime} \neq q, \forall l^{\prime}$. In order to remove the IBI, we need to ensure that the BEM coefficients corresponding to all BEM frequencies for a particular path $l$ should lie on independent estimation bases (or subspaces), i.e., the columns $\mathbf{f}_{q}(i)$ in $\mathbf{F}$ are orthogonal to one another. From the orthogonality of the columns $\mathbf{f}_{q}(i)^{H} \mathbf{f}_{q^{\prime}}(i)=0\left(q \neq q^{\prime}\right)$, we obtain

$$
\begin{array}{r}
e^{\left(\omega_{q_{2}}-\omega_{q_{1}}\right)\left(n_{1}+i\right)}+\ldots+e^{\left(\omega_{q_{2}}-\omega_{q_{1}}\right)\left(n_{P}+i\right)}=0, \\
q_{1}, q_{2} \in\{0, \ldots, Q\}, q_{1} \neq q_{2} .
\end{array}
$$

For various BEM models, the BEM frequencies $\omega_{q}$ 's are fixed and (23) is not satisfied. This leads to IBI during BEM estimation whose amount may be significant when the pilot power increases. In order to satisfy (23), in Section V-B, we propose to adjust the pilot positions $n_{p}$ so that the estimation bases become nearly orthogonal and IBI becomes close to zero. Furthermore, it can be verified that the condition (23) is related to (18), as shown in Section IV-B.

Remark 1. From (21) and (23), note that for perfect IBI cancelation, a minimum number of sub-blocks (equivalently, pilot clusters) is required, i.e., $P \geq(Q+1)$. For high mobility channels (large Doppler $f_{\max }$ ) and large transmission block length $N, Q$ can be large, and accordingly, many pilot clusters are required to perfectly cancel the IBI, leading to lower efficiency.

Assuming that the feasibility condition $P \geq(Q+1)$ in Remark 1 is satisfied, and the IBI becomes zero in (22), only the IPI term remains as the interfering term in the estimation of $h_{q}(l)$. Next, it can be noted that the BEM coefficient for a particular $q$ and $l$, i.e., $h_{q}(l)$, is contained in $M$ pilot equations only. For example, the variable $h_{q}(0)$ is contained in the first $M$ equations $\mathbf{y}(i), i=0, \ldots, M-1$. Collecting all the equations which contain $h_{q}(0)$ for $i=0, \ldots, M-1$, we get

$$
\begin{aligned}
& \mathbf{y} \triangleq\left[\mathbf{f}_{q}^{*}(0) \mathbf{y}(0), \mathbf{f}_{q}^{*}(1) \mathbf{y}(1), \ldots, \mathbf{f}_{q}^{*}(M-1) \mathbf{y}(M-1)\right]^{T} \\
& =P \underbrace{\left[\begin{array}{cccc}
b_{L} & 0 & \ldots & 0 \\
b_{L+1} & b_{L} & \ldots & 0 \\
b_{L+2} & b_{L+1} & \ldots & 0 \\
\vdots & \vdots & \ddots & \vdots \\
b_{L+M-1} & b_{L+M-2} & \ldots & b_{L+(M-L-1)^{+}}
\end{array}\right]}_{\mathcal{B}}\left[\begin{array}{c}
h_{q}(0) \\
h_{q}(1) \\
h_{q}(2) \\
\vdots \\
h_{q}(L)
\end{array}\right]
\end{aligned}
$$

From (24), it is clear that the coefficient $h_{q}(0)$ can be estimated interference-free if the first column of $\mathcal{B}$ is uncorrelated with all other columns (Similarly, all other $h_{q}(l), l \neq 0$, can also be estimated). Specifically, the following equations should be satisfied.

$$
\sum_{i=L}^{L+M-\tau-1} b_{i+\tau}^{*} b_{i}=0, \tau=1, \ldots, L .
$$

By analyzing the IPI during the estimation of other BEM coefficients, we get the same set of equations as above (details omitted here). (25) implies that $\mathbf{B}_{i}^{H} \mathbf{B}_{i}$ in (17) is a diagonal 
matrix, and therefore, (17) is satisfied. It is worth noting that the above equation (25) suggests a pilot sequence with impulselike aperiodic autocorrelation properties. This motivates us to use Huffman sequence as the pilot sequence (for CE) in each sub-block of the transmission frame. An interesting property of Huffman sequence is that its aperiodic autocorrelation is almost impulse-like with zero side-lobes at all time-shifts except at the last one [36], i.e., exactly the set of conditions for interferencefree BEM estimation as mentioned in (25). Please refer to Section V-A where we explicitly discuss the Huffman sequence based pilot designs.

\section{B. Structure of the $\boldsymbol{\Phi}^{H} \boldsymbol{\Phi}$ Matrix in (16)}

Once we choose Huffman sequence as the pilot sequence, (25) is satisfied. Equivalently, (17) is satisfied, and thus the sub-matrices ${ }^{3}$ (see (16) and Fig. 3), i.e., $\boldsymbol{\Psi}_{q_{1} q_{1}}\left(q_{1}=0, \ldots, Q\right)$, lying on the main diagonal of $\boldsymbol{\Phi}^{H} \boldsymbol{\Phi}$ become the scaled identity matrix $\mathcal{P} \mathbf{I}_{L+1}$. Also, the magnitudes of the off-diagonal elements of the off-diagonal $(L+1) \times(L+1)$ sub-matrices $\boldsymbol{\Psi}_{q_{1} q_{2}}, q_{1} \neq q_{2}$ become significantly small. We can observe this by expanding $\boldsymbol{\Psi}_{q_{1} q_{2}}$ in (26) (shown on top of next page), where we denote $\Delta_{q_{1} q_{2}} \triangleq \omega_{q_{2}}-\omega_{q_{1}}$, and $u=(m-n)$. Then, the value of $\left[\boldsymbol{\Psi}_{q_{1} q_{2}}\right]_{(m, n)}$ without the external summation in (26) can be written as

$$
\begin{aligned}
& e^{j \Delta_{q_{1} q_{2}}\left(n_{p}\right)} \sum_{i=|u|}^{M-1}\left(b_{L+i-u^{+}}^{*} b_{L+i-(-u)^{+}}\right) e^{j \Delta_{q_{1} q_{2}}\left(i+m-u^{+}-1\right)} \\
& \stackrel{(a)}{\approx} e^{j \Delta_{q_{1} q_{2}}\left(n_{p}\right)} \sum_{i=|u|}^{M-1} b_{L+i-u^{+}}^{*} b_{L+i-(-u)^{+}} \stackrel{(b)}{=} 0
\end{aligned}
$$

where the approximation $(a)$ is because the phase rotation due to $e^{j \Delta_{q_{1} q_{2}}\left(i+m-u^{+}-1\right)}$ is small for typical values of $\Delta_{q_{1} q_{2}}$ and small values of $i(i \leq M)$, and the equality $(b)$ is due to the aperiodic autocorrelation properties of Huffman sequence as shown in (25). The above approximation is more accurate if the sequence length $M$ is small. Thus the off-diagonal elements of the matrices $\boldsymbol{\Psi}_{q_{1} q_{2}}\left(q_{1} \neq q_{2}\right)$ become significantly small, resulting in near-diagonal $\boldsymbol{\Psi}_{q_{1} q_{2}}$. Finally, the matrix $\boldsymbol{\Phi}^{H} \boldsymbol{\Phi}$ is of the form as shown in Fig. 3.

From Fig. 3, we observe that $\boldsymbol{\Phi}^{H} \boldsymbol{\Phi}$ still contains nonzero elements on the main diagonal of the off-diagonal submatrices which need to be minimized for enhanced channel estimation. For off-diagonal sub-matrix $\boldsymbol{\Psi}_{q_{1} q_{2}}$, the diagonal elements $\left[\boldsymbol{\Psi}_{q_{1} q_{2}}\right]_{(m, m)}(m=1, \ldots, L+1)$ in (26) can be written as

$\left[\boldsymbol{\Psi}_{q_{1} q_{2}}\right]_{(m, m)}=\sum_{i=0}^{M-1} e^{j \Delta_{q_{1} q_{2}}(i+m-1)} b_{L+i}^{*} b_{L+i}\left(\sum_{p=1}^{P} e^{j \Delta_{q_{1} q_{2}} n_{p}}\right)$

Using approximations similar to (27), for typical values of $\left(\Delta_{q_{1} q_{2}}, i\right),\left[\boldsymbol{\Psi}_{q_{1} q_{2}}\right]_{(m, m)}$ is almost the same for all $m=$ $1, \ldots, L+1$, i.e., for all the diagonal elements of $\boldsymbol{\Psi}_{q_{1} q_{2}}$, and therefore, we denote $\beta_{q_{1} q_{2}} \triangleq\left[\boldsymbol{\Psi}_{q_{1} q_{2}}\right]_{(m, m)}, \forall m$. Note that

${ }^{3}$ Here we define the sub-matrices as $\boldsymbol{\Psi}_{q_{1} q_{2}} \triangleq \sum_{i=1}^{P} \mathbf{B}_{i}^{H} \mathbf{D}_{q_{1} i}^{H} \mathbf{D}_{q_{2} i} \mathbf{B}_{i}$. the term in the bracket of (28) is related to (23) which aims for minimum IBI. Thus, minimizing the $(m, m)$-th, i.e., the diagonal term of $\boldsymbol{\Psi}_{q_{1} q_{2}}\left(q_{1} \neq q_{2}\right)$ is equivalent to reducing the IBI during BEM estimation.

\section{BEM-MSE metric approximation}

Based on the above analysis, we present below a simplified BEM-MSE expression. The $\boldsymbol{\Phi}^{H} \boldsymbol{\Phi}$ matrix in (16) can be written as (see Fig. 3)

$$
\begin{aligned}
& \boldsymbol{\Phi}^{H} \boldsymbol{\Phi} \equiv\left[\begin{array}{cccc}
\boldsymbol{\Psi}_{00} & \boldsymbol{\Psi}_{01} & \ldots & \boldsymbol{\Psi}_{0 Q} \\
\boldsymbol{\Psi}_{10} & \boldsymbol{\Psi}_{11} & \ldots & \boldsymbol{\Psi}_{1 Q} \\
\vdots & \ldots & \ddots & \vdots \\
\boldsymbol{\Psi}_{Q 0} & \boldsymbol{\Psi}_{Q 1} & \ldots & \boldsymbol{\Psi}_{Q Q}
\end{array}\right] \\
& \approx\left[\begin{array}{cccc}
\mathcal{P} \mathbf{I}_{L+1} & \beta_{01} \mathbf{I}_{L+1} & \ldots & \beta_{0 Q} \mathbf{I}_{L+1} \\
\beta_{01}^{*} \mathbf{I}_{L+1} & \mathcal{P} \mathbf{I}_{L+1} & \ldots & \beta_{0 Q-1} \mathbf{I}_{L+1} \\
\vdots & \vdots & \ddots & \vdots \\
\beta_{0 Q}^{*} \mathbf{I}_{L+1} & \beta_{01}^{*} \mathbf{I}_{L+1} & \ldots & \mathcal{P} \mathbf{I}_{L+1}
\end{array}\right] \\
& =\underbrace{\left[\begin{array}{cccc}
\mathcal{P} & \beta_{01} & \cdots & \beta_{0 Q} \\
\beta_{01}^{*} & \mathcal{P} & \cdots & \beta_{0 Q-1} \\
\vdots & \vdots & \ddots & \vdots \\
\beta_{0 Q}^{*} & \beta_{01}^{*} & \cdots & \mathcal{P}
\end{array}\right]}_{\widetilde{\mathbf{B}}} \otimes \mathbf{I}_{L+1} \equiv \widetilde{\mathbf{B}} \otimes \mathbf{I}_{L+1} .
\end{aligned}
$$

Using (15) and (30), and assuming that $h_{q}(l)$ are i.i.d. with variance $\delta \triangleq 1 /(Q+1)(L+1)$, i.e., $\boldsymbol{\Gamma}=\delta \mathbf{I}_{(Q+1)(L+1)}$, and $\sigma_{v}^{2}=1$ without loss of generality, we get the simplified approximate BEM-MSE expression as follows.

$$
\begin{aligned}
& M S E \approx \operatorname{tr}\left[\left(\delta^{-1} \mathbf{I}_{(Q+1)(L+1)}+\widetilde{\mathbf{B}} \otimes \mathbf{I}_{L+1}\right)^{-1}\right] \\
& =\operatorname{tr}\left[\left(\widehat{\mathbf{B}} \otimes \mathbf{I}_{L+1}\right)^{-1}\right]=\operatorname{tr}\left[\widehat{\mathbf{B}}^{-1} \otimes \mathbf{I}_{L+1}\right]=(L+1) \operatorname{tr}\left[\widehat{\mathbf{B}}^{-1}\right],
\end{aligned}
$$

where $\widehat{\mathbf{B}} \triangleq\left(\delta^{-1} \mathbf{I}_{Q+1}+\widetilde{\mathbf{B}}\right)$.

Remark 2. Note that $\boldsymbol{\Phi}^{H} \boldsymbol{\Phi}$ in (15) is of dimension $(Q+$ $1)(L+1) \times(Q+1)(L+1)$, and thus its inversion during numerical search for optimal pilot parameter (specifically, pilot position) is computationally quite expensive (of the order $\left.\mathcal{O}\left((Q+1)^{3}(L+1)^{3}\right)\right)$. On the other hand, the simplified BEM-MSE in (31) involves $\widehat{\mathbf{B}}$ of dimension $(Q+1) \times(Q+1)$ only. Thus, the simplified BEM-MSE expression allows us to optimize the pilot parameters with much lower complexity, which will be used to serve as a benchmark against another low-complexity algorithm proposed in Section V.

Remark 3. For the impulse pilot, although the off-diagonal elements of the sub-matrices $\boldsymbol{\Psi}_{q_{1} q_{2}}\left(q_{1} \neq q_{2}\right)$ are zeros (i.e. (25) is satisfied because $b_{i+L}=0, i \neq 0$ ), the main-diagonal is still prominent, leading to IBI and poor CE. Also, impulse pilot tends to suffer from high PAPR, and is therefore, not preferred. 


$$
\Psi_{q_{1} q_{2}}=\sum_{p=1}^{P} e^{j \Delta_{q_{1} q_{2}} n_{p}}\left[\begin{array}{cccc}
\sum_{i=0}^{M-1} e^{j \Delta_{q_{1} q_{2}}} b_{L+i}^{*} b_{L+i} & \sum_{i=1}^{M-1} e^{j \Delta_{q_{1} q_{2}}} b_{L+i}^{*} b_{L+i-1} & \cdots & \sum_{i=L}^{M-1} e^{j \Delta_{q_{1} q_{2} i} b_{L+i}^{*} b_{i}} \\
\sum_{i=1}^{M-1} e^{j \Delta_{q_{1} q_{2}}{ }^{i}} b_{L+i-1}^{*} b_{L+i} & \sum_{i=0}^{M-1} e^{j \Delta_{q_{1} q_{2}}(i+1)} b_{L+i}^{*} b_{L+i} & \cdots & \sum_{i=L-1}^{M-1} e^{j \Delta_{q_{1} q_{2} i} b_{L+i}^{*} b_{i+1}} \\
\vdots & \vdots & \ddots & \vdots \\
\sum_{i=L}^{M-1} e^{j \Delta_{q_{1} q_{2}}{ }^{2}} b_{i}^{*} b_{L+i} & \sum_{i=L-1}^{M-1} e^{j \Delta_{q_{1} q_{2}}(i+1)} b_{i+1}^{*} b_{L+i} & \cdots & \sum_{i=0}^{M-1} e^{j \Delta_{q_{1} q_{2}}(i+L)} b_{L+i}^{*} b_{L+i}
\end{array}\right]
$$

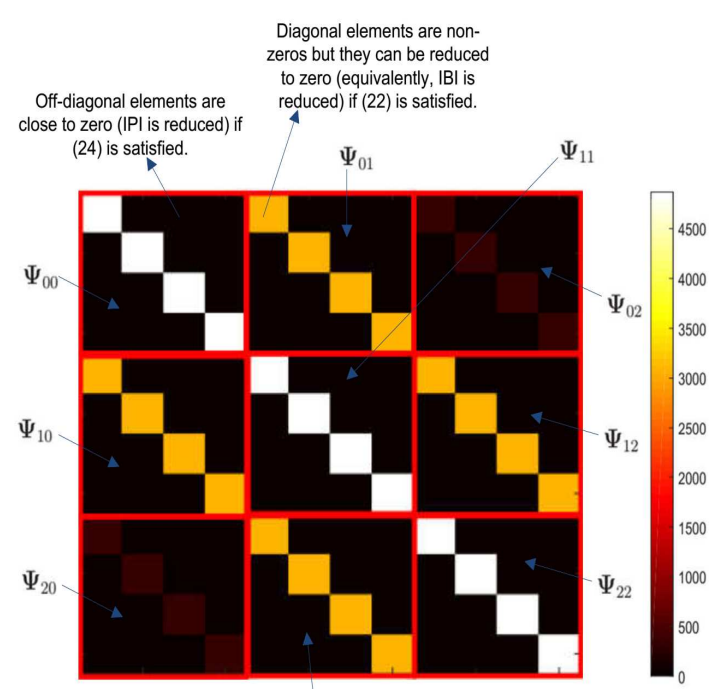

$\boldsymbol{\Psi}_{21}$
Fig. 3: An example of $\left|\boldsymbol{\Phi}^{H} \boldsymbol{\Phi}\right|$ matrix showing the interference during BEM coefficients' estimation, where $Q=2, L=3$. Periodic pilot placement is adopted. Because of this, the $\Psi_{01}, \Psi_{10}, \Psi_{12}, \Psi_{21}$ sub-matrices have large undesired values (yellow-colored) on their main diagonals. The off-diagonal elements of the sub-matrices $\boldsymbol{\Psi}_{q_{1} q_{2}}, q_{1} \neq q_{2}$, are close to zero due to the aperiodic autocorrelation property of Huffman sequences.

\section{Pilot DESIGN}

As discussed in Section IV, minimizing the MSE is equivalent to solving (17) and (18). Based on our above analysis, it is required to solve (25) and (23), respectively. To this end, we consider a two-step approach. In the first step (Section V-A), we propose to solve (25) using a Huffman sequence which has zero aperiodic auto-correlation sidelobes at all the non-zero shifts except at the last one. In the second step (Section V-B), we propose a low-complexity pilot placement strategy to satisfy the IBI condition (23).

\section{A. Huffman sequence design}

We propose to solve the first condition (25) using Huffman sequence, thanks to its impulse-like aperiodic autocorrelation property. Specifically, for a length- $N$ Huffman sequence, zero autocorrelation sidelobes can be observed up to the first $(N-2)$ time shifts [36]. This implies that for a DSC with $L$ multipaths, a Huffman sequence of length at least $(L+2)$ is required in order to satisfy (25). For example, for $L=3$, we need a length-5 Huffman sequence, say $\mathbf{b}_{1 \times 5}$, and the overall pilot cluster, together with zero-padding as shown in (4), is given as $\left[\mathbf{0}_{1 \times 3} \mathbf{b}_{1 \times 5} \mathbf{0}_{1 \times 3}\right]$. Note that it is possible to find multiple Huffman sequences to get (25) satisfied. The issue here is to select the one with very low PAPR.
The Huffman sequence generation, summarized in Algorithm 1, is described as follows. Consider a polynomial $P=\prod_{n=0}^{N-2}\left(x-\phi_{n} r_{n}\right)$, where the root $r_{n}=\alpha^{n}$ [36], with $\alpha=e^{j 2 \pi /(N-1)}$, and the radius $\phi_{n}$ can take a value of either $t$ or $1 / t$ for a real value $t$. For a given $t, \mathcal{C}=2^{N-1}$ such polynomials, denoted as $\left\{P_{1}, \ldots, P_{\mathcal{C}}\right\}$, are possible. A Huffman sequence $\mathbf{g}_{i, t}$ is obtained corresponding to each polynomial $P_{i}$ by extracting its coefficients. For a given $t$, the sequence with the lowest PAPR is then obtained as $\mathbf{g}_{t}=\arg \min _{i=1, \ldots, C} \rho\left(\mathbf{g}_{i, t}\right)$ (Step 5). Next, we vary the value of $t$, and choose the Huffman sequence with the lowest PAPR as $\mathbf{g}^{\star}=\arg \min _{t} \rho\left(\mathbf{g}_{t}\right)$ (Step 7).

Finally, we note that the energy ratio of a Huffman sequence is large (equivalently, PAPR is less) when about half of the roots $r_{n}$ lie on circle of radius $t$ and the other half on circle of radius $1 / t$ [36]. Thus, in Step 2, we need to form the polynomials for those cases in which $\phi_{n}=t$ only for $\left\lceil\frac{N-1}{2}\right\rceil$ out of $N-1$ roots, whereas $\phi_{n}=1 / t$ for rest of the roots. As a result, for every $t$, only $\mathcal{C}=\left(\begin{array}{c}N-1 \\ {[N-1 / 2\rceil}\end{array}\right)$ Huffman sequences need to be obtained, thereby reducing the search complexity, as compared to the exhaustive search where $\mathcal{C}=2^{N-1}$.

In our present work, where the number of multi-paths $L$ is not large, Algorithm 1 is sufficient for searching lowPAPR Huffman sequences of small lengths. However, for large Huffman sequences, even after choosing $\mathcal{C}=\left(\begin{array}{c}N-1 \\ \lceil N-1 / 2\rceil\end{array}\right)$, and after taking into account the invariance operation of the roots [37], the search complexity increases exponentially with the sequence length. For such cases, a low-complexity algorithm can be employed with non-exhaustive search space, as suggested in [37]. If we want to obtain real-valued Huffman sequences for odd $N$, we should also take into account the extra roots $r_{n}=\alpha^{n} e^{j \pi /(N-1)}$ for polynomial generation [37] (see Step 2 of Algorithm 1). For further details, readers are referred to [36], [37], [43].

\section{B. Pilot placement design}

In this subsection, we optimize the pilot placement to satisfy (23) for reduced IBI. Specifically, we aim to adjust the pilot positions $n_{p}, \forall p$, for orthogonal (estimation) bases in the matrix $\mathbf{F}$ in (21). An interesting observation is that the pilot position optimization can be designed separately from the sequence design.

We consider the following multi-objective optimization 


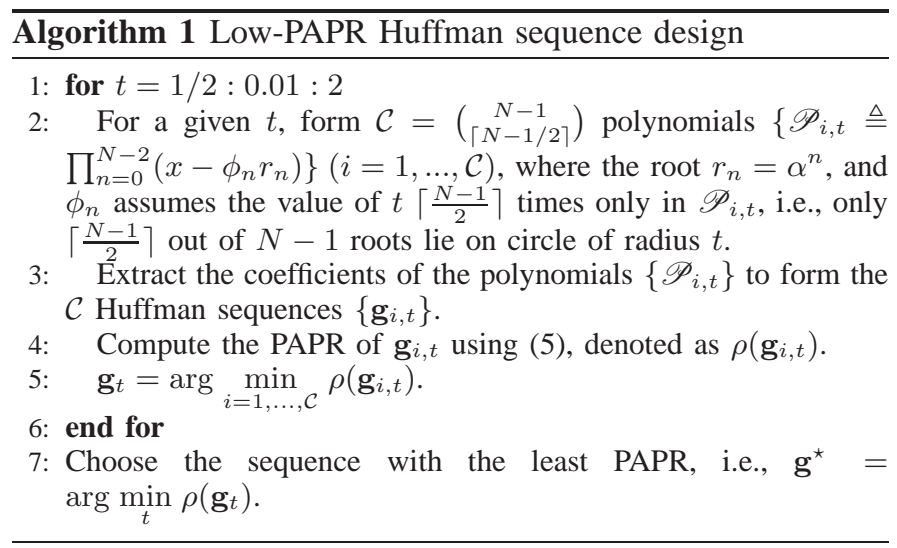

problem.

$$
\min _{n_{p} \in \mathcal{N} \forall p}\left|\sum_{p=1}^{P} e^{j \Delta_{q_{1} q_{2}} n_{p}}\right|, \forall \Delta_{q_{1} q_{2}}, q_{1}, q_{2} \in\{0, \ldots, Q\}, q_{1} \neq q_{2},
$$

$$
\begin{array}{ll}
\text { s.t. } & \left|n_{p}-n_{p^{\prime}}\right| \geq(2 L+M), p \neq p^{\prime}, \\
& n_{P}+M=(N-L+1),
\end{array}
$$

where $\mathcal{N}$ denotes the full search space of the pilot positions, i.e., $\mathcal{N}=\{1, \ldots, N\}$. Note that by solving (P1), the magnitude of the left-hand-side of (23) will be minimized. The first inequality constraint implies that the "starting" non-zero pilot symbols of two different pilot clusters should be separated by at least $(2 L+M)$ (see Fig. 2 for reference), where overlapping of zeros of two adjacent pilot clusters is not allowed. The second equality constraint implies that the transmission block should end with a pilot sequence followed by $L$ zeros, thus avoiding inter-block interference to the next block.

On careful examination of the sub-matrices $\boldsymbol{\Psi}_{q_{1} q_{2}}$, we find that only $Q$ objectives need to be minimized in (P1). Moreover, in our channel model, the BEM frequencies are equi-spaced, i.e., $\Delta:=\Delta_{q_{1} q_{2}}=\omega_{q_{2}}-\omega_{q_{1}}=\omega_{q_{3}}-\omega_{q_{2}}$, and so on. Finally, we can re-formulate (P1) as a min-max problem as follows.

$$
n_{p}^{\star}=\arg \min _{n_{p} \in \mathcal{N} \forall p} \max _{\kappa \in\{1, \ldots, Q\}}\left|\sum_{p=1}^{P} e^{j \kappa \Delta n_{p}}\right|,
$$

s.t. Constraints in (P1).

The rationale of (P2) is to minimize the maximum correlation between the estimation subspaces in (21). Intuitively, it makes sense that for minimum inter-BEM frequency interference during BEM estimation, the subspaces associated with the pilot sampling positions must be minimally correlated. Note that in the conventional periodic sampling by pilots, the estimation subspaces are highly correlated which leads to severe performance degradation in BEM estimation. Note also that by solving (P2), the diagonal elements of $\boldsymbol{\Psi}_{q_{1} q_{2}}$ get reduced in magnitude, thereby diagonalizing the $\boldsymbol{\Phi}^{H} \boldsymbol{\Phi}$ matrix.
Indeed, it is observed through our simulations that the proposed pilot position optimization problem (P2) works remarkably well for the moderate to high normalized Doppler scenarios.

The optimization problem (P2) is a combinatorial optimization problem. For small $Q$ (e.g. $Q=2$ ) and small $N$ values, numerical search may be used to find the nearoptimal pilot positions. One low-complexity approach is to search for $n_{p} \forall p$ in a search space around the periodic pilot positions [29]. However, for large $Q$ (high Doppler scenario) and large $N$, numerical search can be computationally very expensive, and therefore, stochastic search methods such as sequential/parallel stochastic search [44] or discrete stochastic optimization [23] are necessitated to find good pilot positions in a low-complexity manner. Thus, for large $N$ and large $Q$ values, we resort to the stochastic sequential search (SSS) algorithm (see Algorithm 2) to solve (P2).

Let us denote the objective in (P2) as $f(\mathbf{n}) \triangleq$ $\max _{\kappa \in\{1, \ldots, Q\}}\left|\sum_{p=1}^{P} e^{j \kappa \Delta n_{p}}\right|$, where $\mathbf{n}=\left\{n_{1}, n_{2}, \ldots, n_{P}\right\}$ denotes the pilot position vector. In Algorithm 2, I initial pilot positions (each pilot position vector denoted by $\mathbf{n}$ as shown in Step 3), are generated randomly in the outer loop, keeping in mind the pilot separation constraints in (P1). For each initial $\mathbf{n}$, the first non-zero position of each pilot cluster, i.e., $n_{p}(p=1, \ldots, P)$ is sequentially updated one after another while the positions of all other pilot clusters $n_{p^{\prime}}, p^{\prime} \neq p$ are kept fixed. After updating $n_{p}$, the new pilot vector $\mathbf{n}_{p}$ is given as

$$
\begin{aligned}
\mathbf{n}_{p}=\arg & \min _{\widetilde{\mathbf{n}}} f(\widetilde{\mathbf{n}}), \\
\text { s.t. } & \widetilde{\mathbf{n}}\left(p^{\prime}\right)=\mathbf{n}\left(p^{\prime}\right), p^{\prime} \neq p ; \widetilde{\mathbf{n}}(p) \in \mathcal{N} \backslash\left\{n_{p^{\prime}}, p^{\prime} \neq p\right\}, \\
& \text { Constraints in }(\mathrm{P} 1) .
\end{aligned}
$$

Step 5 in Algorithm 2 ensures that the sequential update should stop when the solution is trapped in a local minimum. Similarly, for each of the $I$ random position initializations, the best local solution is found and stored. Out of these $I$ good solutions, the final solution is obtained by simply choosing the one with the minimum $f(\mathbf{n})$.

Although the SSS method does not analyze all the pilot positions exhaustively, its performance is found to be significantly better than the conventional periodic sampling approach. Moreover, our approach, based on exact analysis of the MSE equations, gives us useful insights into the design of pilot positions.

Remark 4. It should be noted that if we wish to transmit data with large efficiency on high Doppler channels, $Q\left(2\left\lceil f_{\max } N T\right\rceil\right)$ can be large and sufficient number of pilot clusters may not be available (i.e., $P<Q+1$, see Remark 1). In such a case, solving (P2) directly may not give good pilot placement results. This is because when $P<Q+1$, some of the columns of $\mathbf{F}$ (see (21)) will always be highly correlated. Although solving (P2) may, in general, de-correlate the columns of $\mathbf{F}$, most of the columns will still be highly correlated, leading to significant IBI during BEM estimation. Instead, we suggest to minimize the maximum correlation 


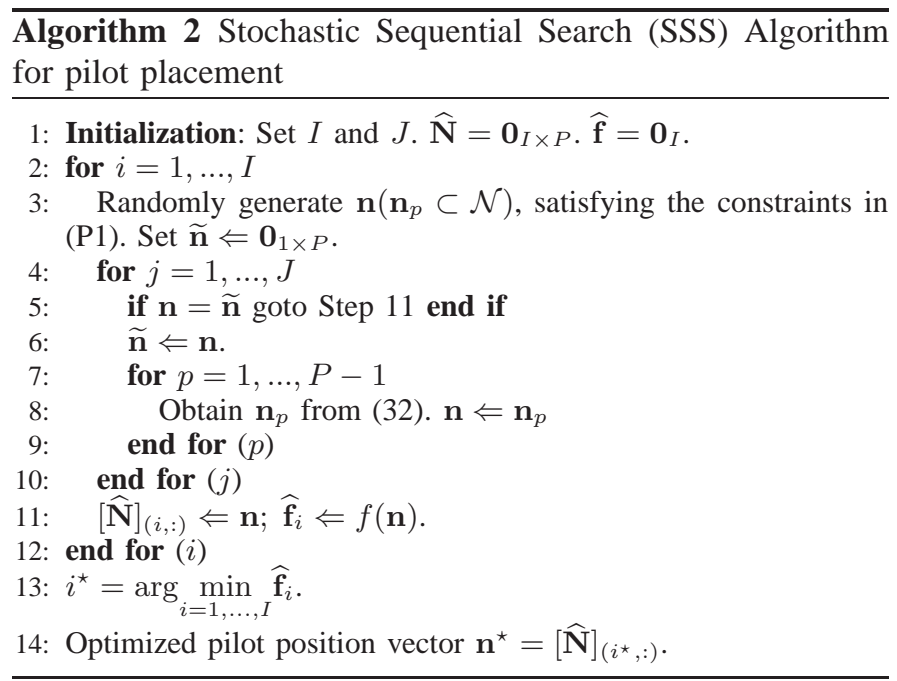

between only the first few columns of F. Specifically, $\kappa$ in (P2) should assume a maximum value of $(P-1)$, i.e., $\kappa \in\{1, \ldots,(P-1)\}$, so that at least $P$ columns become uncorrelated to a large extent. Indeed, this approach shows remarkable performance improvement after pilot placement, as also verified through our simulations.

\section{Complexity of pilot placement design}

Notice that the pilot placement search complexity can be prohibitively high using the exhaustive search method. In particular, for searching $P$ optimal pilot positions (with the design constraints as mentioned in $(\mathrm{P} 2)$ ) in a length- $N$ block, approximately $\left(\begin{array}{l}N \\ P\end{array}\right)$ searches would be required. For example, searching 9 pilot positions in a block of length $N=459$ as in Fig. 12 settings (see Section VI), about $10^{18}$ searches are required. This search complexity is reduced drastically using our proposed pilot placement strategy, as explained next.

Based on the exponential BEM, we have obtained the optimization function $f(\mathbf{n}) \triangleq \max _{\kappa \in\{1, \ldots, Q\}}\left|\sum_{p=1}^{P} e^{j \kappa \Delta n_{p}}\right|$ (see (P2)). Clearly, the main complexity in computing $f(\mathbf{n})$ comes from $P$ complex addition operations for each of the $Q$ different $\kappa^{\prime} s$, and the max. operation which is essentially a comparison operation with a complexity of $\mathcal{O}(Q)$, totaling to a complexity of $\mathcal{O}(P Q+Q)) \sim \mathcal{O}(P Q)$ operations.

Now, within each inner iteration $(j=1, \ldots, J)$ of the SSS (see Algorithm 2), $\mathbf{n}_{p}$ computation (Step 8) involves $\mathcal{O}(N)$ $f(\mathbf{n})$ calculations and $\mathcal{O}(N)$ comparisons for the minimization operations in (32). Thus, $\mathbf{n}_{p}$ computation involves $\mathcal{O}(N)+$ $N \mathcal{O}(P Q) \sim \mathcal{O}(N P Q)$ operations. Since $\mathbf{n}_{p}$ is computed for $(P-1)$ pilot positions, and assuming that the inner and outer iterations occur up to $J$ (Step 4) and $I$ (Step 2) times, respectively, the overall complexity is given by $I J \mathcal{O}\left(P^{2} Q N\right)$ operations.

Note that a significant complexity reduction in our approach comes by considering the objective function $f(\mathbf{n})$ which involves only $\mathcal{O}(P Q)$ addition operations. On the other hand, other works such as [17], [38] assume a direct MSE objective minimization for each of the pilot positions, which involves a complexity of $\mathcal{O}\left(Q^{3}\right)$ complex multiplication operations because of the matrix inversion during the MSE computation. Furthermore, the genetic algorithm based pilot position optimization in [38] is generally more complex compared to our Algorithm 2, and may involve tuning of various hyperparameters as well as partial a priori knowledge of the solution.

It is worth noting that sometimes the pilot placement may result in highly uneven (e.g., very large) data sub-blocks. Although this improves the overall MSE performance, the bit error rate (BER) improvement may be limited, especially for the zero-forcing $(\mathrm{ZF})$ or minimum-sum mean square error (MMSE) equalizers. This is because a long data sub-block implies that the pilot symbols on both sides of the data subblock may not be able to sample the time-varying channel fast enough to catch up with its short coherence interval, leading to localized CE errors over individual long data subblock. Therefore, we use trellis-based maximum-likelihood (ML) equalizers for data equalization to achieve good BER performance. Finally, in our simulations, we use the pilot position search using simplified BEM-MSE (see (31)) minimization as a benchmark to assess the performance of our proposed pilot optimization problem (P2).

\section{Simulation RESUlts}

In this section, we carry out numerical simulation to evaluate the proposed pilot design for channel estimation in DSC. We consider a DSC of order $L=3$, i.e., four multipaths. Each channel tap is modeled as an i.i.d. random variable correlated in time according to Jakes' model with the correlation function given as $J_{0}\left(2 \pi n f_{\max } T\right)$, where $J_{0}(\cdot)$ is the zeroth-order Bessel function of the first kind [6]. The average channel gain for each path is assumed to be $\frac{1}{L+1}$ so that the overall channel gain is unity. Unless stated exclusively, the signal to noise ratio (SNR) is defined as the average SNR (averaged over all the data and the pilot sub-blocks in the transmission frame), i.e., $\rho \triangleq \frac{\mathcal{P}_{T}}{(N-2 L P) \sigma_{v}^{2}}$ [2], where $\mathcal{P}_{T}$ is the total power over the entire transmission block of the sequencepilot based scheme, $\sigma_{v}^{2}$ is the noise variance, and $P$ is the number of sub-blocks within the transmission frame. We make sure the number of pilots is sufficient so that the number of equations is not less than $(Q+1)(L+1)$, the number of unknown BEM coefficients. Unless stated otherwise, the transmission efficiency $\eta$ is assumed to be $2 / 3$, i.e., the data symbols constitute $66.67 \%$ of the transmission frame. In our simulations, the CE MSE for the $l^{\text {th }}$ multi-path is defined as

$$
M S E_{l}=\frac{1}{M N} \sum_{m=1}^{M} \sum_{n=0}^{N-1}\left|h^{(m)}(n ; l)-\widehat{h}^{(m)}(n ; l)\right|^{2},
$$

where $h^{(m)}(n ; l)$ and $\widehat{h}^{(m)}(n ; l)$ denote the actual and estimated channels, respectively, for the $m^{t h}$ transmission block, $M$ the number of Monte-Carlo simulations, and $\widehat{h}^{(m)}(n ; l)=$ $\sum_{q=0}^{Q} \widehat{h}_{q}^{(m)}(l) e^{j \omega_{q} n}$, with $\widehat{h}_{q}^{(m)}(l)$ being the $m^{\text {th }}$ block's estimat- 
ed BEM coefficient corresponding to the BEM frequency $\omega_{q}$ (see (14)).

First, we consider the case where the normalized Doppler spread $f_{\max } T$ for the fading channel is 0.005 . Since $L=3$, the Huffman sequence should be of length $M=(L+2)=5$. Due to $L$ zero-padding on both sides of the sequence, the total pilot length in a pilot sub-block is $2 L+5=11$. In order to maintain $\eta=2 / 3$ for the periodic pilot placement, the data length is set to be 22 for each sub-block. Considering $P=3$ sub-blocks in the transmission frame, the overall frame length is $N=99$ symbols, and thus the number of BEM coefficients, $Q=2\left\lceil f_{\max } N T\right\rceil=2$.

Using Algorithm 1, we find the lowest PAPR real-valued $^{4}$ Huffman sequence-based pilot to be $[0,0,0,1,1,0.5,-1,1,0,0,0]$. The positions of the first non-zero symbols of the periodic pilot sequence in the 3 subblocks are given as $\left[n_{1}^{(o)}=26, n_{2}^{(o)}=59, n_{3}^{(o)}=92\right]$. During pilot position optimization, in order to avoid inter-block interference at the end of the current block (in other words, the second constraint in (P1) is satisfied), we let the last sub-block's first non-zero pilot position to be $n_{3}=92$, i.e., same as periodic placement case ${ }^{5}$. Using numerical search to solve (P2), high quality pilot position solutions are obtained as $\left[n_{1}^{\star}=8, n_{2}^{\star}=60, n_{3}^{\star}=92\right]$. The pilot positions before and after optimization are shown in Fig. 4. Note that after optimization, the data length is different in each sub-block (unlike periodic placement case where each sub-block has data length of 22). Finally, the pilot positions obtained by optimizing the simplified BEM-MSE in (31) are given as $\left[n_{1}^{\star}=8, n_{2}^{\star}=48, n_{3}^{\star}=92\right]$.

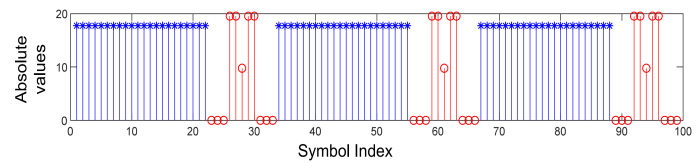

(a)

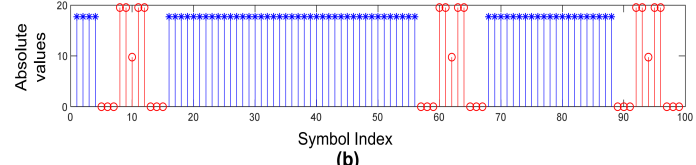

(b)

Fig. 4: Absolute values of the data (blue asterisks) and pilot (red circles) symbols in a transmission frame of length $N=99$ with (a) Periodic pilot positions (b) Pilot positions after optimization

Fig. 5 shows the channel MSE performances of various algorithms. We also compare our sequence-pilot based CE results with the impulse-pilot based scheme, i.e., the case when impulse pilot ("TDKD" pilot structure, see Fig. 1) is used in all the 3 sub-blocks for CE, each consisting of 14 data symbols and 7 (one impulse pilot surrounded by $L=3$ zeros on either side) pilot symbols, totaling to a block length ${ }^{6} N=63$.

Note that for the above mentioned comparison, power fairness should be carefully taken into account for the sequence

\footnotetext{
4 For length-5 Huffman sequence, we note that the lowest PAPR attained by both the complex and real-valued Huffman sequences are the same. ${ }^{5}$ Note that for the case of non-contiguous packet-based transmission, even the last sub-block's pilot position can be optimized, eventually leading to 4 data sub-blocks and 3 pilot sub-blocks. ${ }^{6}$ Note that block length $N=63$ symbols is chosen in order to maintain $\eta=2 / 3$.
}

and impulse pilot based schemes. Specifically, for CE, the total pilot power in a transmission block is assumed to be the same in both the schemes. Similarly, for BER performances, we ensure that the power per data or information symbol is exactly the same in both the schemes. For our simulations, power allocation between the data and the pilots is denoted by the pilot-to-data-ratio (PDR), defined as

$$
\begin{aligned}
& \text { PDR } \triangleq \frac{\text { Total pilot power in a block }}{\text { Total data power in a block }} \\
& =\frac{\text { Total pilot power in a block }}{\text { number of data symbols in a block } \times \text { power per data symbol }} .
\end{aligned}
$$

As discussed previously, for the transmission efficiency $\eta=$ $2 / 3$, the sequence pilot scheme has 66 data symbols whereas the impulse pilot scheme has only 42 data symbols in a block. Therefore, when the total pilot power and the power per data symbol are kept the same in both these schemes, the PDRs of the two schemes can be different. For example, for the simulations in Figs. 5 and 6, PDRs for impulse and sequence pilot schemes are approximately 0.37 and 0.23 , respectively.

In Figs. 5 and 6, "NCS-CE-BEM" denotes the noncritically-sampled CE-BEM which provides the best BEM fit for the CE-BEM [17], and "CE-BEM" denotes the criticallysampled CE-BEM mentioned in [2]. "Impulse", "Huffman", and "Random" denote impulse pilot, proposed Huffmansequence based pilot, and random pilot sequence, respectively. "Periodic placement" and "Optimized placement" mean that the pilot placements are periodic and the proposed optimized pattern, respectively. "P-BEM" denotes the polynomial BEM [18]. "GCE-BEM" denotes the generalized CE-BEM [3] in which oversampling is used to obtain a more precise channel model. Note that in all the schemes except "GCE-BEM", $Q=2$, and therefore $P=(Q+1)=3$ sub-blocks are sufficient for BEM estimation. On the other hand, in "GCEBEM", $Q=4$ due to oversampling, and at least 5 sub-blocks should be used, resulting in the frame length of $N=105$ (comparable to $N=99$ for the sequence-based proposed schemes). Finally, "NCS-CE-BEM-MSE, optimized" denotes the performances obtained by optimizing the pilot positions based on the simplified BEM-MSE metric in (31).

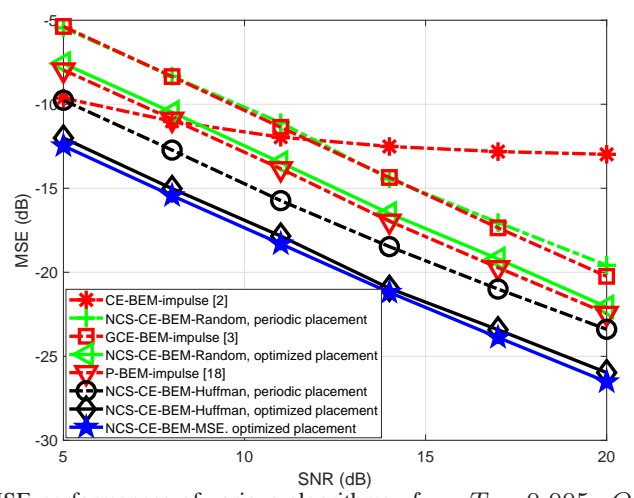

Fig. 5: MSE performances of various algorithms. $f_{\max } T=0.005, Q=2$.

From Fig. 5, it can be observed that for the same pilot power in all the schemes, the Huffman sequence pilots with periodic 
pilot placement ("NCS-BEM-Huffman, periodic placement") provide much better $\mathrm{CE}$, as compared to the CE-BEM and GCE-BEM based impulse pilot schemes. Notably, the performance of the P-BEM based impulse pilot scheme is quite close to the Huffman pilot scheme, but the later has the added advantage of low PAPR. Due to the pilot power spread over the sequence symbols, the Huffman pilot cluster has a much lower PAPR of 2.59 (please refer to (5) for PAPR calculation.) as opposed to 7 for the impulse pilot. It is worth noting that for the "NCS-CE-BEM-Huffman, periodic placement", only the condition (25) is satisfied due to the Huffman sequence but the IBI values (see (23)) are large as the pilot positions are not optimized here. On the other hand, the proposed "NCS-CEBEM-Huffman, optimized placement" satisfies (25) and tends to minimize IBI also in (23), and thus provides significantly better CE MSE compared to both the impulse as well as the Huffman pilots with periodic pilot placement. Specifically, its MSE performance is close to the benchmark simplified BEMMSE (see (31)) optimization based pilot positions.

We also show random pilot sequence based MSE plots which, as expected, perform poorly as compared to the proposed pilot design. Note that the "Random, optimized placement" scheme shows poor performance although optimized pilot positions are adopted. This is because (25) cannot be satisfied by the random pilot sequence, thus resulting in IPI during BEM estimation which degrades its MSE and BER performances.

Fig 6 shows the BER performances of the various algorithms for QPSK modulation using ML (Viterbi) data equalization. For both the sequence and impulse pilot based schemes, same power is allocated to each data or information symbol, and here, the SNR on the x-axis denotes the SNR per data symbol only. Similar to the trend displayed in the MSE curves shown in Fig. 5, the proposed pilot scheme "NCSCE-BEM-Huffman, optimized placement" shows the best BER performance (close to the benchmark scheme "NCS-CE-BEMMSE, optimized placement").

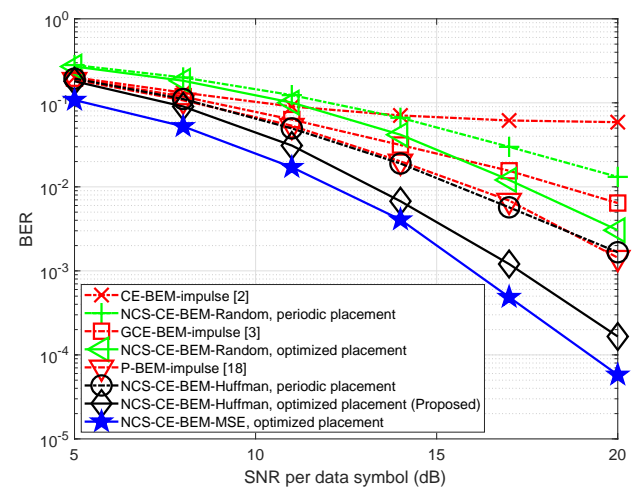

Fig. 6: BER performances of various algorithms, QPSK modulation, $f_{\max } T=$ $0.005, Q=2$.

Note that in Figs. 5 and 6, "MSE-optimized placement" has been used as the benchmark. In this case, we refer to the pilot position optimization based on the approximate MSE expression of the BEM coefficients' estimation, as mentioned in Section IV-C. Fig. 7 shows that the actual BEM-MSE (15) and the approximate BEM-MSE (31) are quite close for both the periodic as well as the optimized pilot placement cases. This validates the approximate BEM-MSE expression in (31), and thus the pilot position optimization based on the approximate BEM-MSE is also justified.

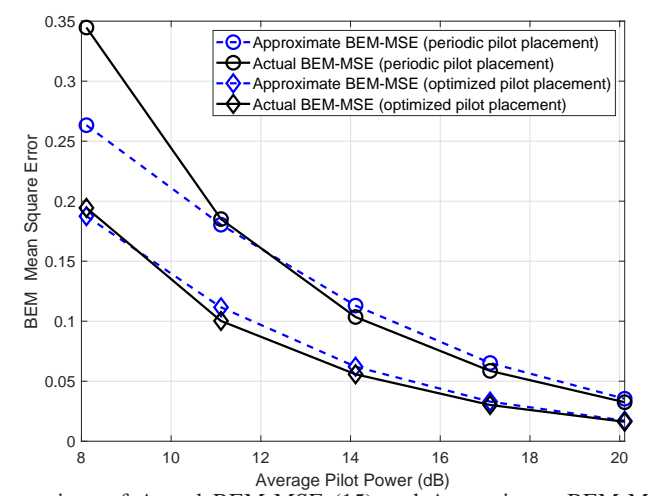

Fig. 7: A comparison of Actual BEM-MSE (15) and Approximate BEM-MSE (31) at different values of Average pilot SNRs; $f_{\max } T=0.005, Q=2$.

Next, we consider higher normalized Doppler $f_{\max } T=$ 0.01 with $L=3$ multi-paths and block length $N=165$. As a result, $Q=4$, and we need 5 pilot clusters for 5 sub-blocks. Here, since our main focus is low-PAPR transmission, from now onwards, we consider sequence-based transmission only. For the periodic placement, each sub-block has 33 symbols, with 22 data and 11 pilot symbols (length-11 sequence), leading to 110 data symbols and 55 pilot symbols in the entire frame. However, after pilot placement optimization, the pilot positions become irregular (no longer periodic), and therefore, each sub-block's data length also changes. The proposed pilot cluster consists of the same length-11 Huffman sequence as earlier. The PDR is set to 0.234 , and other channel parameters are the same as earlier.

The periodic pilot placement is given by $\left[n_{1}^{(o)}=26, n_{2}^{(o)}=\right.$ $\left.59, n_{3}^{(o)}=92, n_{4}^{(o)}=125, n_{5}^{(o)}=158\right]$, whereas the proposed pilot placement solution (obtained from Algorithm 2) is obtained as $\left[n_{1}^{(\star)}=8, n_{2}^{(\star)}=45, n_{3}^{(\star)}=82, n_{4}^{(\star)}=119, n_{5}^{(\star)}=\right.$ 158]. Note that after optimization, the first pilot cluster is closer to the edge of the frame, i.e., $n_{1}^{(\star)}=8$. This implies that the channel sampling by the pilots should start from the beginning of the frame otherwise the information in the first few channel samples could not be taken into account (such as in periodic placement case, which begins with data sub-block followed by pilot cluster) for CE. Similarly, a pilot cluster placed closer to the frame end gives better CE.

In Fig. 8, we plot the absolute values of the $\boldsymbol{\Phi}^{H} \boldsymbol{\Phi}$ matrix for $f_{\max } T=0.01$ which also reflects the interference pattern during the BEM estimation. We observe that due to the proposed pilot design, the $\boldsymbol{\Phi}^{H} \boldsymbol{\Phi}$ matrix becomes almost diagonal. This implies that the IBI and IPI are significantly reduced during BEM estimation due to the proposed Huffman sequence and pilot placement, leading to reduced MSE, and therefore better $\mathrm{CE}$ which is further confirmed by Fig. 9.

Fig. 9 shows that the proposed Huffman sequence-based 


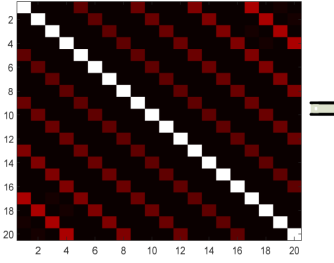

(a) Periodic pilot placement

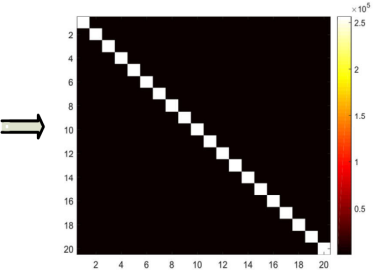

(b) Optimized pilot placement
Fig. 8: $\left|\boldsymbol{\Phi}^{H} \boldsymbol{\Phi}\right|$ matrix due to (a) Periodic pilot positions (b) Pilot positions after optimization; $f_{\max } T=0.01, Q=4$.

optimized pilot design ("Huffman, Optimized placement") has significantly better CE MSE performance (closest to the benchmark "MSE-optimized placement" scheme), as compared to random sequence based pilots and periodic pilot positions. The BER plots in Fig. 10 show similar trends, thus proving the effectiveness of our proposed pilot design.
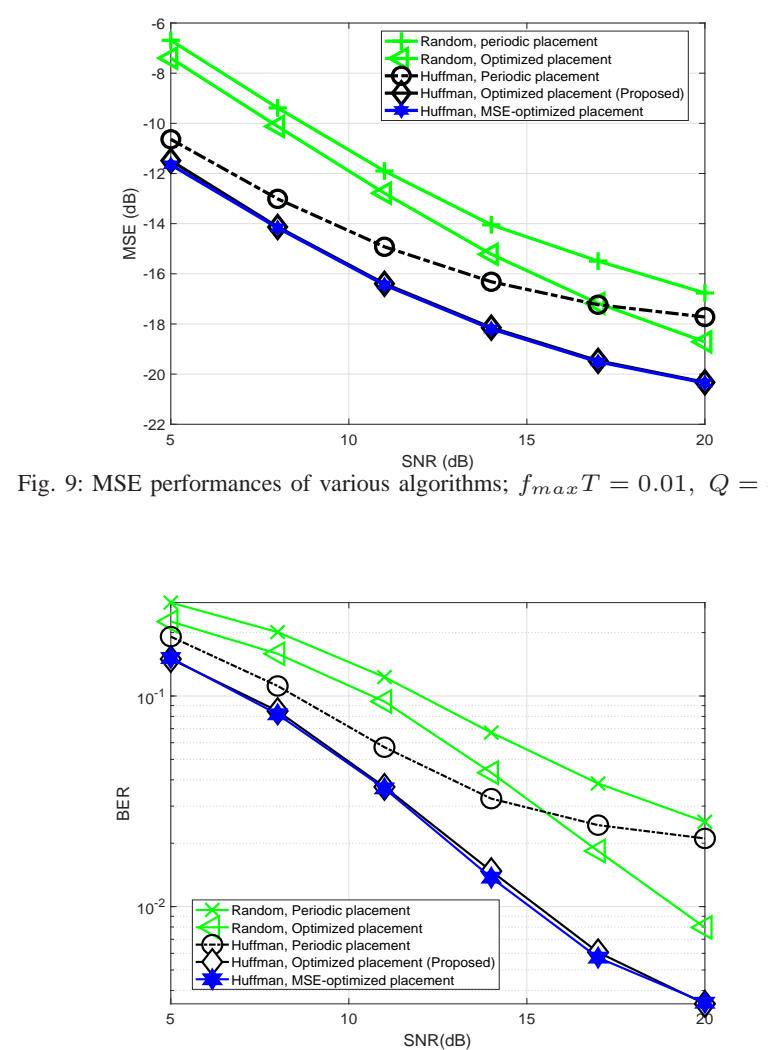

Fig. 10: BER performances of various algorithms, QPSK modulation, $f_{\max } T=$ $0.01, Q=4$.

Remark 5. Note that the "MSE-optimized" scheme serves as a good benchmark for MSE performances of different algorithms when the number of BEM coefficients $Q$ is small. However, for higher Doppler, $Q$ becomes large and hence the "MSE-optimized" scheme needs to invert a larger (albeit much smaller than the original MSE matrix in (15)) $(Q+1) \times(Q+1)$ matrix for each pilot position during exhaustive search, which can be computationally quite expensive. In contrast, our proposed "min-max" search (see (P2)) does not require any matrix inversion, and thus, can be used to find the sub-optimal pilot positions in a low-complexity manner, even for large Doppler and large $Q$.

In Fig. 11, we consider higher normalized Doppler $f_{\max } T=0.02$, with a transmit symbol duration $T=33.33 \mu \mathrm{s}$. Thus, $f_{\max }=600 \mathrm{~Hz}$, which corresponds to a mobility of $324 \mathrm{~km} / \mathrm{hr}$ for a carrier frequency of $2 \mathrm{GHz}$. The efficiency $\eta=59.2 \%$ and the PDR is 0.54 . The channel order is $L=3$, i.e., 4 multi-paths. Accordingly, a low PAPR length- 5 Huffman sequence with $L$ zero-padding (ZP) on both sides $\left[\mathbf{0}_{1 \times 3}, 1,1,0.5,-1,1, \mathbf{0}_{1 \times 3}\right]$ is used as the pilot cluster for $\mathrm{CE}$. We also consider the CE MSE performances of other competitive sequences such as Zadoff-Chu and Generalized Barker sequences [36] which have low PAPR and low OPAAC. For each case, the block length is $N=243$ with 9 subblocks, each comprising a data sub-block of 16 data symbols and a pilot sub-block of 11 pilot symbols ( 5 sequence symbols with $3 \mathrm{ZP}$ on either sides). The sequence pilots are placed periodically within the transmission block because here, we want to analyze only the effect of different sequence types (and not pilot placement) on the CE performance. The number of BEM coefficients $Q=2\left\lceil f_{\max } N T\right\rceil=10$.

Note that although a low-PAPR Huffman sequence is chosen, it has a slightly higher PAPR of 2.58, as compared to the other sequences which have a PAPR of 2.2 (consider the ZP during PAPR calculation, see (5)). However, Huffman sequence outperforms all other sequences in terms of $\mathrm{CE}$, thanks to its exact zero OP-AAC property. Thus, with only a slight compromise in PAPR, Huffman sequence shows better CE capabilities. In comparison, an impulse pilot cluster (not shown here) would have a much higher PAPR of 7 in this case.

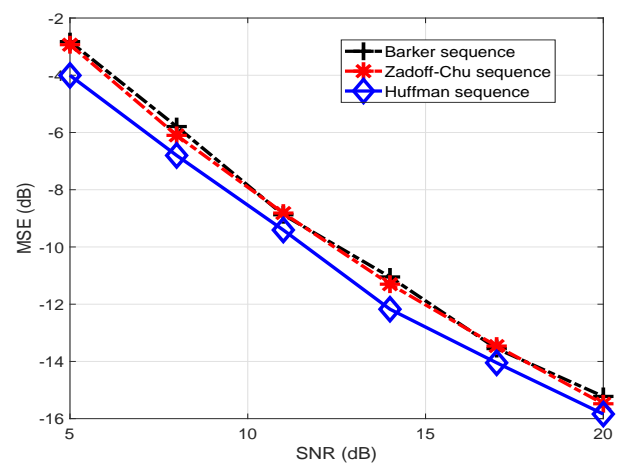

Fig. 11: MSE performances of various sequences; $f_{\max } T=0.02, Q=10, L=3$, number of sub-blocks $P=9$, block length $N=243$, efficiency $=59.2 \%$, Periodic pilot placement.

In Fig. 12, we compare the performances of various sequences for high mobility channels (large $f_{\max }$ ), and larger multi-paths $(L)$ and higher efficiency $(\eta)$, as compared to Fig. 11. The system parameters are: $T=16.67 \mu s, f_{\max }=$ 600 , mobility $v=324 \mathrm{~km} / \mathrm{hr}, \eta=2 / 3, L=5$, sequence length $=(L+2)=7, Q=10$, number of sub-blocks $P=9$, block length $N=459$, and the pilot positions are optimized using Algorithm 2. Since $N$ and $f_{\max }$ are quite large, and we wish $\eta=2 / 3$, we use only 9 pilot clusters (sub-blocks) here. Due to length- $L \mathrm{ZP}$ on both sides of the sequence pilot, each of the 9 pilot clusters is of length-17 $(7+5 \times 2=17)$. 
However, here the number of pilot clusters $P<Q$, therefore, due to insufficient number of pilot clusters, BEM coefficients' estimation is not completely interference-free (see Remark 4) which may affect the system BERs also. For such a case, as mentioned in Remark 4, the maximization operation in (P2) will be done only over $(P-1)=8$ terms, i.e., $\kappa \in\{1, \ldots, 8\}$ in $(\mathrm{P} 2)$.

For the above system parameters, Fig. 12 shows the BER performances of various sequence pilot based schemes after pilot position optimziation. Clearly, the Huffman sequence shows the best BER performance, with an SNR gain greater than $3 \mathrm{~dB}$ at a BER of $10^{-2}$, as compared to the other sequence based schemes. Furthermore, from Table I, it can be observed that the PAPR of the Huffman sequence is only slightly higher than the Zadoff-Chu and Barker sequences, but its CE MSE and BER performances are comparatively much better. In comparison, an impulse pilot cluster would have a much higher PAPR of 11 in this case.

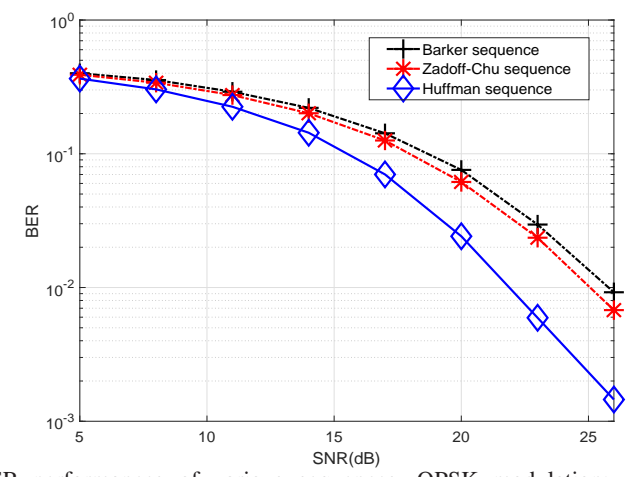

Fig. 12: BER performances of various sequences, QPSK modulation; $f_{\max } T=$ 0.01, $Q=10, L=5$, number of sub-blocks $P=9$, block length $N=459$, efficiency $=66.67 \%$, Proposed pilot placement after optimization.

Finally, in Fig. 13, we consider the performance of our proposed method for the ITU vehicular channel B model with six multi-paths [45]. The symbol duration $T$ is taken to be $2.77 \mu \mathrm{s}$ (equivalently, a bandwidth of $360 \mathrm{KHz}$ ). The carrier frequency $f_{c}=2 \mathrm{GHz}$, mobility $v=162 \mathrm{~km} / \mathrm{hr}$, and the maximum Doppler frequency $f_{\max }=300 \mathrm{~Hz}$. As a result, the normalized Doppler value $f_{\max } T=8.3 \times 10^{-4}$ is very low, and therefore $Q=2$, i.e., only $(Q+1)=3$ BEM coefficients suffice to model the channel. The transmission block length $N$ is taken to be 540 with 489 data symbols and 51 pilot symbols. Thus, a high spectral efficiency $\eta=90.56 \%$ is obtained. It is worth noting that with the above parameters, a block length of $N=540$ is equivalent to six resource blocks (RBs) (2 RBs per slot in a 3-slot LTE frame structure [46]). This corresponds to a total duration of $1.5 \mathrm{~ms}$ frame in a typical LTE physical resource allocation grid [46]. Each block is divided into 3 subblocks, with each sub-block consisting of some data symbols and a length-17 pilot sequence cluster (the pilot sequences are the same as in Fig. 12). $5 \%$ of the total transmission power is allocated to the pilots and the rest to the data symbols. For the conventional (periodic pilot pattern based) scheme, equal number of data symbols, i.e., 163 symbols are placed in each sub-block whereas for our proposed scheme, variable number of data symbols are placed in each sub-block, depending on the pilot pattern optimization.

Fig. 13 shows that our proposed Huffman sequence based optimized pilot pattern indeed achieves better BER performances for the above scenario, as compared to other sequences with conventional pilot placement. Specifically, the SNR gain is about $4 \mathrm{~dB}$ at a BER of $10^{-3}$, as compared to other sequence pilot based schemes.

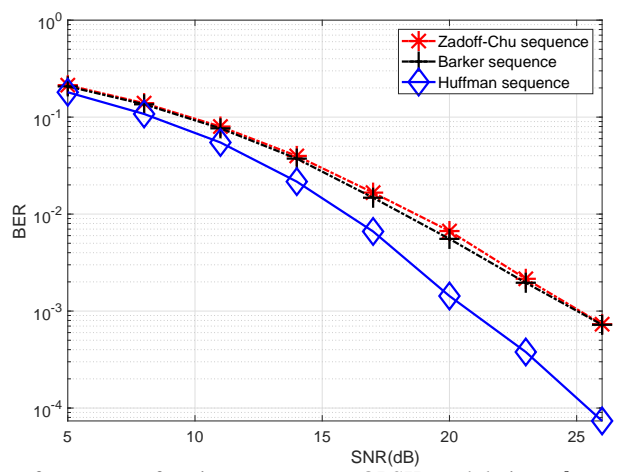

Fig. 13: BER performances of various sequences, QPSK modulation; $f_{\max } T=8.3 \times$ $10^{-4}, Q=2$, six multi-paths, number of sub-blocks $P=3$, block length $N=540$, efficiency $=90.56 \%$.

\section{CONCLUSION}

In this paper, we have investigated sequence-based pilot designs for channel estimation (CE) in doubly-selective channels (DSC). Specifically, we propose the use of low-PAPR Huffman sequences for CE instead of the conventional high-PAPR impulse pilots. We provide a detailed analysis of CE with sequence-based pilots which gives useful insights into the pilot design process. Based on our analysis, we also propose a lowcomplexity pilot placement strategy to significantly improve the CE. Simulation results show that our proposed pilot design significantly outperforms the conventional periodic pilots under various Doppler scenarios, both in terms of CE mean square error and bit error rates.

\section{ACKNOWLEDGEMENTS}

The authors would like to thank the Editor and the anonymous reviewers for their insightful comments and suggestions.

Kushal Anand received his PhD from the School of Electrical and Electronic Engineering (EEE), Nanyang Technological University (NTU), Singapore. Currently, he is a Research Fellow with the School of EEE, NTU. His research interests include transmission techniques for high mobility wireless channels, interference management in wireless networks, and signal processing for communications.

Yong Liang Guan obtained his $\mathrm{PhD}$ degree from the Imperial College of London, UK, and Bachelor of Engineering degree with first class honors from the National University of Singapore. He is a tenured associate professor at the School of Electrical and Electronic Engineering, Nanyang Technological University, Singapore, where he is now the Head of two industry collaboration labs: NTU-NXP Smart Mobility Lab, and Schaeffler Hub for Advanced Research (SHARE) at NTU. His research interests broadly include coding and signal processing for communication systems, data storage systems 
TABLE I: Performances of various pilot sequences at an average SNR of $23 \mathrm{~dB}$

\begin{tabular}{|c|c|c|c|c|c|c|}
\hline Sequence Type & & Length-7 Sequence & $\begin{array}{c}\text { Pilot cluster } \\
\text { for a sub-block }\end{array}$ & CE MSE (dB) & BER & PAPR of pilot \\
\hline Huffman & $\mathbf{b}_{H}=$ & {$\left[\begin{array}{r}1,-0.5506,0.1516+0.9891 i \\
-0.6341-0.5446 i,-0.1516-0.9891 i \\
-0.5506,-1\end{array}\right.$} & {$\left[\mathbf{0}_{1 \times 5}, \mathbf{b}_{H}, \mathbf{0}_{1 \times 5}\right]$} & -20.83 & $5.9 \times 10^{-3}$ & 3.2 \\
\hline Zadoff-Chu & $\mathbf{b}_{Z}=$ & $\begin{array}{r}1,0.6235-0.7818 i,-0.9010-0.4339 i \\
0.6235+0.7818 i,-0.9010-0.4339 i \\
0.6235-0.7818 i, 1\end{array}$ & {$\left[\mathbf{0}_{1 \times 5}, \mathbf{b}_{Z}, \mathbf{0}_{1 \times 5}\right]$} & -18.53 & $2.3 \times 10^{-2}$ & 2.42 \\
\hline Barker & $\mathbf{b}_{B}={ }^{0.645}$ & $\begin{array}{r}+0.7636 i, 0.6133+0.7899 i, 0.6178-0.7863 i \\
0.8087-0.5882 i,-0.2198+0.9755 i \\
0.9998+0.0223 i,-0.8943+0.4474 i\end{array}$ & {$\left[\mathbf{0}_{1 \times 5}, \mathbf{b}_{B}, \mathbf{0}_{1 \times 5}\right]$} & -17.96 & $2.9 \times 10^{-2}$ & 2.42 \\
\hline
\end{tabular}

and information security systems. He is an Editor of the IEEE TRANSACTIONS ON VEHICULAR TECHNOLOGY, and was an AE of the IEEE SIGNAL PROCESSING LETTER. His homepage is at http://www.ntu.edu.sg/home/eylguan/.

Xiaobei Liu received the B.S degree from Fudan University, Shanghai, China in 1998, and the Ph.D. degree from Nanyang Technological University (NTU), Singapore, in 2004. From 1998 to 2000, she was an Engineer with Datang Mobile Communications Equipment Co., Ltd. and from 2007 to 2010 she was a Senior Digital Signal Processing Engineer in Wireless Sound Solutions Pte. Ltd. She is currently a Senior Research Scientist in Temasek Laboratories@NTU. Her research interests include signal processing in wireless communications, modulation/coding techniques, blind signal detection and secured communications.

Zilong Liu is a Lecturer at the School of Computer Science and Electronic Engineering, University of Essex, UK, since December 2019. From January 2018 to November 2019, he was a Senior Research Fellow at the Institute for Communication Systems (ICS), Home of the 5G Innovation Centre (5GIC), University of Surrey, UK. From July 2008 to January 2018, he was with the School of Electrical and Electronic Engineering, Nanyang Technological University (NTU), Singapore, first as a Research Associate and since November 2014 a Research Fellow. From Aug. 2009 to Aug. 2013, he was a PhD candidate (part-time) working on "Perfect- and Quasi- Complementary Sequences" advised by Prof. Yong Liang Guan. He received his M.S. degree in the Department of Electronic Engineering from Tsinghua University, and his B.S. degree in the School of Electronics and Information Engineering from Huazhong University of Science and Technology (HUST), China, in 2007 and 2004, respectively. He has been an Associate Editor of IEEE Access since January 2017. He is generally interested in coding and signal processing for various communication systems, with emphasis on signal/waveform design and algebraic coding, error correction codes, iterative receiver design, robust/efficient multiple access communications, and physical layer implementation/prototyping of communications systems. Details of his research can be found at: https://sites.google.com/site/zilongliu2357

Yang Yang received the B.S. and M.S. degrees from Hubei University,Wuhan, China, in 2005 and 2008, respectively, and the $\mathrm{Ph} . \mathrm{D}$. degree from Southwest Jiaotong University, Chengdu, China, in 2013. Since 2013, he has been with the School of Mathematics, Southwest Jiaotong University, where he is currently an Associate Professor. His research interest includes sequence design and its applications.

Zhengchun Zhou received the B.S. and M.S. degrees in mathematics and the Ph.D. degree in information security from Southwest Jiaotong University, Chengdu, China, in 2001, 2004, and 2010, respectively. From 2012 to 2013, he was a Postdoctoral Member with the Department of Computer Science and Engineering, Hong
Kong University of Science and Technology. From 2013 to 2014, he was a Research Associate with the Department of Computer Science and Engineering, the Hong Kong University of Science and Technology. Since 2001, he has been with the Department of Mathematics, Southwest Jiaotong University, where he is currently a Professor. His research interests include sequence design and coding theory. Dr. Zhou was the recipient of the National Excellent Doctoral Dissertation Award in 2013 (China).

Pingzhi Fan (M'93-SM'99-F'15) received his MSc degree in computer science from the Southwest Jiaotong University, China, in 1987, and $\mathrm{PhD}$ degree in Electronic Engineering from the Hull University, UK, in 1994. He is currently a distinguished professor and director of the institute of mobile communications, Southwest Jiaotong University, China, and a visiting professor of Leeds University, UK (1997-), a guest professor of Shanghai Jiaotong University (1999-). He is a recipient of the UK ORS Award (1992), the NSFC Outstanding Young Scientist Award (1998), IEEE VTS Jack Neubauer Memorial Award (2018), and 2018 IEEE SPS Signal Processing Letters Best Paper Award. His current research interests include vehicular communications, massive multiple access and coding techniques, etc. He served as general chair or TPC chair of a number of international conferences including VTC 2016Spring, IWSDA 2019, ITW 2018 etc. He is the founding chair of IEEE Chengdu (CD) Section, IEEE VTS BJ Chapter and IEEE ComSoc CD Chapter. He also served as an EXCOM member of IEEE Region 10, IET(IEE) Council and IET Asia Pacific Region. He has published over 300 international journal papers and 8 books (incl. edited), and is the inventor of 25 granted patents. He is an IEEE VTS Distinguished Lecturer (2015-2019), and a fellow of IEEE, IET, CIE and CIC.

Erry Gunawan received the BSc degree in electrical and electronic engineering from the University of Leeds, the MBA and $\mathrm{PhD}$ degrees, both from Bradford University. From 1984 to 1988, he worked as a Satellite Communication System Engineer at Communication Systems Research Ltd, Ilkley, UK. In 1988, he moved to Space Communication (SAT-TEL) Ltd, Northampton, UK. He joined the School of Electrical and Electronic Engineering, Nanyang Technological University, in 1989, and currently, he is an associate professor in the same school. He has been a consultant to Sytek Technical Associates, to Addvalue Communications Pte Ltd, and to RFNet Technologies Pte Ltd, Singapore. He has published more than 150 papers in International Journals and conferences on error correction codings, modeling of cellular communications systems, power control for CDMA cellular systems, MAC protocols, multicarrier modulations, multiuser detections, space-time coding, radio-location systems, MIMO interference channel, and the applications of UWB radar for vital sign sensing and medical imaging.

\section{REFERENCES}

[1] J. Wu and P. Fan, "A survey on high mobility wireless communications: Challenges, opportunities and solutions," IEEE Access, vol. 4, pp. 450476, Jan. 2016 
[2] X. Ma, G. B. Giannakis, and S. Ohno, "Optimal training for block transmissions over doubly selective wireless fading channels," IEEE Trans. Signal Process., vol. 51, no. 5, pp. 1351-1366, May 2003.

[3] G. Leus, "On the estimation of rapidly time-varying channels," in Proc. IEEE European signal Processing Conference (EUSIPCO), Sept. 2004, pp. 2227-2230.

[4] F. Qu and L. Yang, "On the estimation of doubly-selective fading channels," IEEE Trans. Wireless Commun., vol. 9, no. 4, pp. 1261-1265, Apr. 2010.

[5] J. K. Tugnait, S. He, and H. Kim, "Doubly selective channel estimation using exponential basis models and subblock tracking," IEEE Trans. Signal Process., vol. 58, no. 3, pp. 1275-1289, Mar. 2010.

[6] S. He and J. K. Tugnait, "On doubly selective channel estimation using superimposed training and discrete prolate spheroidal sequences," IEEE Trans. Signal Process., vol. 56, no. 7, pp. 3214-3228, Jul. 2008.

[7] J. K. Tugnait and S. He, "Doubly-selective channel estimation using datadependent superimposed training and exponential basis models," IEEE Trans. Wireless Commun., vol. 6, no. 11, pp. 3877-3883, Nov. 2007.

[8] Z. Tang, R. C. Cannizzaro, G. Leus, and P. Banelli, "Pilot-assisted timevarying channel estimation for OFDM systems," IEEE Trans. Signal Process., vol. 55, no. 5, pp. 2226-2238, May 2007.

[9] H. C. Lee, C. W. Chen, and S. W. Wei, "Channel estimation for OFDM system with two training symbols aided and polynomial fitting," IEEE Trans. Commun., vol. 58, no. 3, pp. 733-736, Mar. 2010.

[10] T. Zemen, L. Bernado, N. Czink, and A. F. Molisch, "Iterative timevariant channel estimation for $802.11 \mathrm{p}$ using generalized discrete prolate spheroidal sequences," IEEE Trans. Veh. Technol., vol. 61, no. 3, pp. 1222-1233, Mar. 2012.

[11] P. S. Rossi and R. R. Muller, "Slepian-based two-dimensional estimation of time-frequency variant MIMO-OFDM channels," IEEE Signal Process. Lett., vol. 15, pp. 21-24, Jan. 2008.

[12] D. Hu, X. Wang, and L. He, "A new sparse channel estimation and tracking method for time-varying OFDM systems," IEEE Trans. Veh. Technol., vol. 62, no. 9, pp. 4648-4653, Nov. 2013.

[13] Y. Liao, G. Sun, X. Shen, S. Zhang, X. Yang, X. Zhang, H. Yao, and N. Zhang, "Bem-based channel estimation and interpolation methods for doubly-selective ofdm channel," in 2018 IEEE International Conference on Smart Internet of Things (SmartIoT), Aug. 2018, pp. 70-75.

[14] H. Senol, E. Panayirci, and H. V. Poor, "Nondata-aided joint channel estimation and equalization for OFDM systems in very rapidly varying mobile channels," IEEE Trans. Signal Process., vol. 60, no. 8, pp. 42364253, Aug. 2012.

[15] I. Barhumi and M. Moonen, "MLSE and MAP equalization for transmission over doubly selective channels," IEEE Trans. Veh. Technol., vol. 58, no. 8, pp. 4120-4128, Oct. 2009.

[16] Z. Sheng, H. D. Tuan, H. H. Nguyen, and Y. Fang, "Pilot optimization for estimation of high-mobility OFDM channels," IEEE Trans. Veh. Technol., vol. 66, no. 10, pp. 8795-8806, Oct. 2017.

[17] T. Whitworth, M. Ghogho, and D. McLernon, "Optimized training and basis expansion model parameters for doubly-selective channel estimation," IEEE Trans. Wireless Commun., vol. 8, no. 3, pp. 14901498, Mar. 2009

[18] H. Hijazi and L. Ros, "Polynomial estimation of time-varying multipath gains with intercarrier interference mitigation in ofdm systems," IEEE Trans. Veh. Technol., vol. 58, no. 1, pp. 140-151, Jan 2009.

[19] H. Hijazi and L. Ros, "Joint data QR-detection and Kalman estimation for OFDM time-varying Rayleigh channel complex gains," IEEE Trans. Commun., vol. 58, no. 1, pp. 170-178, Jan. 2010.

[20] D. K. Borah and B. T. Hart, "Frequency-selective fading channel estimation with a polynomial time-varying channel model," IEEE Trans. Commun., vol. 47, no. 6, pp. 862-873, Jun. 1999.

[21] T. Zemen and C. F. Mecklenbrauker, "Time-variant channel estimation using discrete prolate spheroidal sequences," IEEE Trans. Signal Process., vol. 53, no. 9, pp. 3597-3607, Sept. 2005.

[22] P. Suárez-Casal, J. A. García-Naya, L. Castedo, and M. Rupp, "KLTbased estimation of rapidly time-varying channels in MIMO-OFDM systems," in Proc. IEEE Signal Processing Advances in Wireless Communications (SPAWC), Jun. 2013, pp. 654-658.

[23] B. Gong, L. Gui, Q. Qin, X. Ren, and W. Chen, "Block distributed compressive sensing-based doubly selective channel estimation and pilot design for large-scale MIMO systems," IEEE Trans. Veh. Technol., vol. 66, no. 10, pp. 9149-9161, Oct. 2017.

[24] F. Gao, Y. Zeng, A. Nallanathan, and T. Ng, "Robust subspace blind channel estimation for cyclic prefixed MIMO ODFM systems: algorithm, identifiability and performance analysis," IEEE J. Sel. Areas Commun., vol. 26, no. 2, pp. 378-388, Feb. 2008.

[25] S. Park, B. Shim, and J. W. Choi, "Iterative channel estimation using virtual pilot signals for MIMO-OFDM systems," IEEE Trans. Signal Process., vol. 63, no. 12, pp. 3032-3045, Jun. 2015.

[26] A. Saci, A. Al-Dweik, A. Shami, and Y. Iraqi, "One-shot blind channel estimation for OFDM systems over frequency-selective fading channels," IEEE Trans. Commun., vol. 65, no. 12, pp. 5445-5458, Dec. 2017.

[27] F. E. Dorcheh and S. Shahbazpanahi, "Jointly optimal pre- and postchannel equalization and distributed beamforming in asynchronous bidirectional relay networks," IEEE Trans. Signal Process., vol. 65, no. 17, pp. 4593-4608, Sep. 2017.

[28] S. Alireza Banani and R. G. Vaughan, "OFDM with iterative blind channel estimation," IEEE Trans. Veh. Technol., vol. 59, no. 9, pp. 42984308, Nov. 2010

[29] K. Anand, Y. L. Guan, Z. Liu, Y. Yang, Z. Zhou, P. Fan, and E. Gunawan, "Pilot design for channel estimation in doubly selective channel," in 2018 IEEE International Conference on Communication Systems (ICCS), Dec. 2018, pp. 236-241.

[30] A. Goldsmith, Wireless Communications. Cambridge University Press, 2005.

[31] J. Ji, G. Ren, and H. Zhang, "PAPR reduction of SC-FDMA signals via probabilistic pulse shaping," IEEE Trans. Veh. Technol., vol. 64, no. 9, pp. 3999-4008, Sep. 2015

[32] K. Kim, H. Park, and H. M. Kwon, "Optimum clustered pilot sequence for OFDM systems under rapidly time-varying channel," IEEE Trans. Commun., vol. 60, no. 5, pp. 1357-1370, May 2012.

[33] K. M. Z. Islam, T. Y. Al-Naffouri, and N. Al-Dhahir, "On optimum pilot design for comb-type OFDM transmission over doubly-selective channels," IEEE Trans. Commun., vol. 59, no. 4, pp. 930-935, Apr. 2011.

[34] P. Duan, M. He, and M. Xue, "Channel estimation method for timevarying channel based on periodic PN sequence for OFDM systems," in 2009 4th IEEE Conference on Industrial Electronics and Applications, May 2009, pp. 1796-1800.

[35] M. Basaran, H. Senol, S. Erküçük, and H. A. Çirpan, "Channel estimation for TDS-OFDM systems in rapidly time-varying mobile channels," IEEE Trans. Wireless Commun., vol. 17, no. 12, pp. 8123-8135, Dec. 2018.

[36] P. Fan and M. Darnell, Sequence design for communications applications. Research Studies Press, 1996.

[37] L. Bomer and M. Antweiler, "Long energy efficient Huffman sequences," vol. 4, Apr. 1991, pp. 2905-2908.

[38] Z. Tang and G. Leus, "Time-multiplexed training for time-selective channels," IEEE Signal Process. Lett., vol. 14, no. 9, pp. 585-588, Sept. 2007.

[39] F. Hlawatsch and G. Matz, Wireless communications over rapidly time varying channels. Academic Press, 2011.

[40] G. Taubock, F. Hlawatsch, D. Eiwen, and H. Rauhut, "Compressive estimation of doubly selective channels in multicarrier systems: Leakage effects and sparsity-enhancing processing," IEEE J. Sel. Signal Process., vol. 4, no. 2, pp. 255-271, April 2010.

[41] S. Hu, Z. Liu, Y. L. Guan, W. Xiong, G. Bi, and S. Li, "Sequence design for cognitive CDMA communications under arbitrary spectrum hole constraint," IEEE J. Sel. Areas Commun., vol. 32, no. 11, pp. 19741986, Nov. 2014

[42] S. Ohno and G. B. Giannakis, "Capacity maximizing MMSE-optimal pilots for wireless OFDM over frequency-selective block rayleigh-fading channels," IEEE Trans. Inf. Theory, vol. 50, no. 9, pp. 2138-2145, Sept. 2004.

[43] M. H. Ackroyd, "Synthesis of efficient Huffman sequences," IEEE Trans. Aerosp. Electron. Syst., vol. AES-8, no. 1, pp. 2-8, Jan. 1972.

[44] C. Qi, G. Yue, L. Wu, Y. Huang, and A. Nallanathan, "Pilot design schemes for sparse channel estimation in OFDM systems," IEEE Trans. Veh. Technol., vol. 64, no. 4, pp. 1493-1505, Apr. 2015.

[45] R. Jain, "Channel models: A tutorial," http://www.cse.wustl.edu/jain/cse574-08/ftp/channel_model_tutorial.pdf.

[46] “3GPP TS 36.211 v13.2.0 (2016-06)," 3rd Generation Partnership Project; Technical Specification Group Radio Access Network; Evolved Universal Terrestrial Radio Access (E-UTRA); Physical channels and modulation (Release 13), 2016. 